\title{
Taxes and Money in Incomplete Financial Markets
}

\author{
Elena Laureana del Mercato*and Antonio Villanacci ${ }^{\dagger}$
}

September 2, 2004

\begin{abstract}
We analyze a model with incomplete financial markets, where money is needed to pay taxes.

In the case of general tax functions, equilibria exist and are generically locally unique. If the tax function is piecewise linear, tipically, there exists a redistribution of money among households in the first period only which leads to a Pareto superior equilibrium. The intervention does not require either closing markets or upper bounds on the number of households.
\end{abstract}

Key words: taxes, money, incomplete financial markets, general equilibrium, Pareto improvements.

JRL classification numbers: D52, D60, E50, H20.

*Dipartimento di Scienze Economiche e Statistiche, Università degli Studi di Salerno, Via Ponte don Melillo I - 84084 Fisciano (SA), Italy; edmercat@unisa.it.

†Corresponding author. Dipartimento di Matematica per le Decisioni, Universita' degli Studi di Firenze, via Lombroso 6/17, 50134 Firenze, Italy; villanac@unifi.it 


\section{Introduction}

The literature on general equilibrium with incomplete markets analyzed three main properties of equilibria: existence, (generic local) uniqueness or indeterminacy, and Pareto optimality - in the form of generic inefficiency and effectiveness of Pareto improving planner intervention.

Most of the existing contributions studies models without (outside) money. In this paper, we present a model with money and we investigate the above mentioned three problems. ${ }^{1}$

In a classical general equilibrium model with or without complete markets, all trades takes place between the individual and an abstract market."The perfection and simplicity of the trade in the model preclude the role for money as a facilitator of transactions." (Starr (1989)) . In the absence of "imperfections" which prevent people to barter goods, it is impossible to model money in a consistent way. Quoting from Cass and Shell (1980),

"...It is obvious (and well known) that money cannot have a positive price - that is, cannot be a store of value - in a conventional finite horizon-model in which the "end of the world" is known with certainty. The reason is simple. At the end of the last period, money is worthless. Therefore, in the next-to-last period, all individuals desire to dispose of money in order to avoid capital losses. This drives the price of money to zero at the end of the next-to-last period. And so on. Individual with foresight, not wanting to be stuck with the monetary "hot potato", thus drive the price of money to zero in each period."

In the recent literature, several ways have been chosen to solve the above mentioned "hot potato" problem. ${ }^{2}$

First of all, one could assume an infinite horizon model characterized either by overlapping-generations or infinitely-lived consumers.

If one insists in keeping a model with a finite number of periods, some alternatives are possible. The simplest one is to make money another argument of the consumers' utility function, a device which basically denies the very nature of the puzzle of giving positive price to a "worthless" commodity.

Another approach consists in imposing a cash-in-advance constraint. The idea, due to Clower (1967), amounts to introduce an external agent who exchanges goods for money and money for goods ${ }^{3}$; the act of buying and the act of selling are separated and every exchange must involve transactions of money.

Finally, following Knapp (1905) and Lerner (1947), one can assume that in the first period of time, consumers are endowed with some money by the government, and in the last period they have to pay taxes using money.

Observe that both the cash-in-advance and the tax models seem to replace a problem (hot potato for consumers) by another one (hot potato for the "govern-

\footnotetext{
${ }^{1}$ As explained below, we analyze the case in which money is used to pay taxes. In a companion paper, we consider the case of cash-in-advance models - see del Mercato and Villanacci (in progress).

${ }^{2}$ For a discussion of recent contributions on this topic, see Starr (1989) and Magill and Quinzii (1996).

${ }^{3} \mathrm{~A}$ formal analysis of the model is presented in Magill and Quinzii (1992) and (1996).
} 
ment"). As Magill and Quinzii (1996, page 443) say "If money has no value to agents in the private sector, why should it have any value to the government?".

A natural answer consistent with a favorable view of the role of government is that money can be used for policy purposes. Government introduces money because it can be used to decrease the inefficiency of equilibria in a world with some imperfection - say incomplete markets. That observation leads to the core of the long-standing debate on neutrality of money: Is the real side of the economy independent of monetary policy? ${ }^{4}$ More precisely, in an a general equilibrium model with incomplete markets, finite horizon and rational expectations, can monetary policy change equilibrium allocations and/or Pareto improve upon them?

The effects of non-monetary policies have been widely studied in the literature.

Geanakoplos and Polemarchakis (1986) have shown that generically, a planner may Pareto improve upon the competitive outcome by assigning asset portfolios, reallocating initial endowments in period zero among households, and closing financial markets. Their proof requires also an upper bound on the number of households.

Citanna, Kajii and Villanacci (1998) have shown that planner intervention is typically successful if she reallocates the numeraire good among all households in period zero and among two households in one state in period one.

Some attention has also been paid to monetary policies.

Magill and Quinzii (1992) analyze an incomplete market model with cashin-advance money and show that generically in endowments and money supply equilibria are locally unique; they also show that if assets markets are incomplete, local changes in money supply translate in a degree of indeterminacy of equilibria equal to the number of states in the second period minus one, as long as the number of household is bigger than the number of assets.

Carosi (2001) analyzes a model with restricted participation, and proportional taxes and shows that a planner can Pareto improve if she can tax one household in each state in the final period of time (and if the number of states there is not smaller than the number of households).

Tirelli (2003) shows that a planner can Pareto improve in a model with a one-good per spot, incomplete markets, production, taxes only on asset returns and no outside money.

The above papers pay for the freedom of trading on financial markets - absent in Geanakoplos and Polemarchakis (1986) with the need to use what could be seen as a policy of "announcement" of taxes and transfers: credibility of such announcement can be questioned.

Can a planner redistribute wealth only in period zero, leave all markets open and Pareto improve?

The present paper analyzes a model with incomplete markets and money

\footnotetext{
${ }^{4}$ For a brief and clear summary of the main aspect of the controversy, see pages 429-430 in Magill and Quinzii (1996).
} 
used to pay taxes ${ }^{5}$ for which the answer to the above question is positive, without imposing any upper bound on the number of households.

Citanna, Polemarchakis and Tirelli (2002) also answer positively to the above question in a standard model with incomplete financial markets. Planner intervention consists in imposing taxes on trades in assets, creating a difference between purchasing and buying prices. That policy is therefore anonymous, in the sense that is not household dependent. An upper bound on the number of households is required in the proof. ${ }^{6}$

Another main reason of interest in a model with incomplete markets and outside money is the analysis of the determinacy (or local uniqueness) of equilibria. It is well-known that if assets pay in abstract units of account, equilibria exhibit a high ${ }^{7}$ degree of indeterminacy. The basic intuition ${ }^{8}$ behind that result is that different prices across states of nature lead to different purchasing power of the nominal asset returns across those states and therefore they lead to changes in the subspaces of income transfers achievable by the available asset structure. ${ }^{9}$

It could be argued that , “... indeterminacy per se presents a severe practical hurdle for the rational expectation hypothesis. In short, is it plausible to maintain that households are capable of concentrating their beliefs (correctly) on one among a surfeit of possible market outcomes?"10. Indeterminacy could also be seen as a virtue of the model. When so many equilibria are possible, there is room for some institute (the government, worker unions, ...) to "guide" the economy towards the choice of a particular equilibrium with desirable properties, without leading to any disruptive change of the functioning of the economy.

Moreover, indeterminacy seems to suggest that there is an important element which is missing in the model. Since "the reason" for indeterminacy is that the future "price level" is not tied down, introducing outside money will eliminate the problem.

While there is some basis for that conjecture, its validity depends on how money is introduced. While in the cash-in-advance and proportional taxes models, equilibria are typically determinate, in the case of lump sum taxes actually equilibria exhibit one more degree of indeterminacy. For the reasons suggested above, we investigate the case of a general tax function and we in fact show

\footnotetext{
${ }^{5}$ Villanacci (1991) and (1993) prove existence in models with money used to pay proportional and lump sum taxes; moreover, generically in endowments in the former model equilibria are locally unique, and in the latter one, equilibria exhibit a degree of real indeterminacy equal to the number of states in the second period.

${ }^{6}$ The number of households has to be smaller or equal than both (the number of assets minus 1) and (the difference between the number of states and the number of assets plus 1).

${ }^{7}$ The degree of real indeterminacy is equal to the number of states in the second period minus one.

${ }^{8}$ The mathematical intuition is simply that in the system of equations defining the equilibrium set, the number of relevant unknowns is bigger than the number of nonredundant equations.

${ }^{9}$ For a discussion of the indeterminacy issue, see Magill and Quinzii (1996) or Villanacci et Al. (2002) and further references there.

${ }^{10}$ Cass (1992), p. 264.
} 
that equilibria are determinate as long as assets pay in units of money.

The paper is organized as follows. In section 2, we describe the set up of the model. In section 3 , we show existence of equilibria and generic regularity for the case of general tax functions - see Assumption 6 and 21, respectively. In section 4 , in the case of piecewise linear tax function, we prove that monetary policy is not neutral: redistributing wealth in the first period can Pareto improve. Finally, the Appendix contains some more technical notation and the proof of the main theorem of section 4 .

\section{Set up of the model}

There are two periods: today (period $t=0)$ and tomorrow (period $t=1$ ). Today there is one state of the world, denoted by $s=0$. Tomorrow, $S$ states of the world are possible. Therefore, the set of all possible state of the world (also called spots) is $\{0,1, \ldots, S\}$ with generic element $s$.

There are $C$ physical commodities or goods in each spot $s$ denoted by superscript $c \in\{1, \ldots, C\}$. Good $c$ in state $s$ is denoted by superscript $s c$. In total, there are $G \equiv C(S+1)$ goods.

There are $H$ households or consumers labelled by subscript $h \in\{1, \ldots, H\} \equiv$ $\mathcal{H}$ with preferences described by utility function $u_{h}: \mathbb{R}_{++}^{G} \rightarrow \mathbb{R}$.

There are $I$ assets or financial instruments labelled by superscript $i \in\{1, \ldots, I\}$.

Outside money differs from all the other goods because it does not enter households' utility functions, it has to be used to pay taxes, it can be stored and it cannot be issued by households, i.e., its individual demand has to be nonnegative.

Time structure is as follows. In period 0 , households receive initial endowments of goods and initial endowment of outside money; they exchange goods, outside money and assets; finally they consume the goods they acquired. Then, uncertainty is resolved and in period 1, one of $S$ possible states of the world occurs. In each of them, households receive other endowments of goods and of outside money, assets pay their yields; households exchange goods and outside money; finally they pay taxes using outside money and consume the goods they acquired. At the end of the world, all money in the economy is taken away by the government.

The notation we use is summarized below.

- $x_{h}^{s c}$ is the consumption of commodity $c$ in state $s$ by household $h ; x_{h}^{s} \equiv$ $\left(x_{h}^{s c}\right)_{c=1}^{C}, x_{h} \equiv\left(x_{h}^{s}\right)_{s=0}^{S} ; x \equiv\left(x_{h}\right)_{h=1}^{H} \in \mathbb{R}_{++}^{G H}$.

- $e_{h}^{s c}$ is the endowment of commodity $c$ in state $s$ owned by household $h$; $e_{h}^{s} \equiv\left(e_{h}^{s c}\right)_{c=1}^{C}, e_{h} \equiv\left(e_{h}^{s}\right)_{s=0}^{S} ; e \equiv\left(e_{h}\right)_{h=1}^{H} \in \mathbb{R}_{++}^{G H}$.

- $m_{h}^{s}$ is the demand of outside money by household $h$ in spot $s$; we assume that households cannot issue outside money, i.e., $m_{h}^{s} \in \mathbb{R}_{+}$for all $h$ and $s ; m_{h} \equiv\left(m_{h}^{s}\right)_{s=0}^{S} ; m^{0} \equiv\left(m_{h}^{0}\right)_{h=1}^{H} ; m \equiv\left(m_{h}\right)_{h=1}^{H} \in \mathbb{R}_{+}^{(S+1) H}$. 
- $e_{h}^{s m}$ is the endowment of outside money owned by household $h$ in state $s$; $e_{h}^{s m} \in \mathbb{R} ; e_{h}^{m} \equiv\left(e_{h}^{s m}\right)_{s=0}^{S} ; e^{m} \equiv\left(e_{h}^{m}\right)_{h=1}^{H} \in \mathbb{R}^{H(S+1)}$.

- $p^{s c}$ is the price of one unit of commodity $c$ in state $s ; p^{s} \equiv\left(p^{s c}\right)_{c=1}^{C}$; $p \equiv\left(p^{s}\right)_{s=0}^{S} \in \mathbb{R}_{++}^{G}$; prices of goods are expressed in units of outside money.

- An asset $i$ is described by an $(S+1)$ dimensional vector $\left(-q^{i}, y^{1, i}, \ldots, y^{S, i}\right)$. $q^{i}$ is the price of asset $i ; q \equiv\left(q^{i}\right)_{i=1}^{I} \in \mathbb{R}^{I}$; prices of assets are expressed in units of outside money. $y^{s i}$ is the yield of asset $i$ in state $s$, expressed in units of outside money; $y^{s} \equiv\left(y^{s i}\right)_{i=1}^{I} \in \mathbb{R}^{I} ; Y \equiv\left[y^{s i}\right]_{s, i} \in M^{S, I}$, the set of $S \times I$ matrices.

- For $s \geq 1, \widehat{t}^{s}: \mathbb{R}^{I} \times \mathbb{R} \times \mathbb{R}_{++}^{C} \times \mathbb{R}_{++}^{C} \times \mathbb{R} \times \mathbb{R}^{I} \rightarrow \mathbb{R}$,

$\widehat{t}^{s}:\left(b_{h}, m_{h}^{0}, p^{s}, e_{h}^{s}, e_{h}^{s m}, y^{s}\right) \longmapsto \widehat{t}^{s}\left(b_{h}, m_{h}^{0}, p^{s}, e_{h}^{s}, e_{h}^{s m}, y^{s}\right)$

is the tax to be paid by household $h$ in state $s ; \widehat{t} \equiv\left(\widehat{t}^{s}\right)_{s=1}^{S} \cdot \widehat{t}^{s}$ is measured in units of money.

- $b_{h}^{i}$ is the demand of asset $i$ by household $h ; b_{h} \equiv\left(b_{h}^{i}\right)_{i=1}^{I} ; b \equiv\left(b_{h}\right)_{h=1}^{H} \in$ $\mathbb{R}^{I H}$.

Assumption 1 For all $h, u_{h}$ is $C^{3}$, differentiably strictly increasing (i.e., $\forall x_{h} \in$ $\mathbb{R}_{++}^{G}, D u_{h}\left(x_{h}\right) \gg 0$ ), differentiably strictly quasi-concave (i.e., $\forall y \in \mathbb{R}^{G} \backslash\{0\}$ and $\forall x_{h} \in \mathbb{R}_{++}^{G}$ such that $D u_{h}\left(x_{h}\right) y=0$, we have $\left.y^{T} D^{2} u_{h}\left(x_{h}\right) y<0\right)^{11}$, and $\forall u \in \mathbb{R}, c l_{\mathbb{R}^{G}}\left\{x_{h} \in \mathbb{R}_{++}^{G}: u_{h}\left(x_{h}\right) \geq u\right\} \subseteq \mathbb{R}_{++}^{G}$.

The set of utility functions of household $h$ which satisfy Assumption 1 is denoted by $\mathcal{U}_{h}$. Moreover, $\mathcal{U} \equiv \times \mathcal{U}_{h}$ is endowed with the topology of the $C^{3}$ uniform convergence on compact sets .

Remark 2 To show existence of equilibria, our strategy of proof requires the utility functions to be $C^{2}$. We need $C^{3}$ utility functions in the proof of Theorem 32.

We make the following assumptions on the financial and tax structure of the model.

Assumption 3 (Incomplete Markets) $I+1<S$.

Assumption 4 (No redundancy) $\operatorname{rank}\left[\mathbf{1}_{S} \mid Y\right]=I+1$.

The set of all matrices $Y \in M^{S, I}$ that satisfy Assumptions 3 and 4 is denoted by $\mathcal{M}$.

Assumption 5 1. $\forall h, e_{h}^{0 m} \geq 0 ; \forall h, s \geq 1, \quad e_{h}^{0 m}+e_{h}^{s m} \geq 0 ;$

2. $\sum_{h=1}^{H} e_{h}^{0 m}>0 ; \quad \forall s \geq 1, \quad \sum_{h=1}^{H}\left(e_{h}^{0 m}+e_{h}^{s m}\right)>0$.

\footnotetext{
${ }^{11}$ In fact, in the proof of section 4 , we need to assume that $\forall x_{h}, D^{2} u_{h}\left(x_{h}\right)$ is negative definite.
} 
Condition 1 insures that households have a non-negative endowment of money in each state - at worse saving some money from state zero to state $s$.

Condition 2 requires some money to be available to pay taxes.

Assumption 6 For each $s \geq 1, \widehat{t}^{s}$ is a continuous function and satisfies the following conditions.

1. $\forall h, s \geq 1 \exists \varepsilon^{s} \in(0,1)$ such that $\forall\left(b_{h}, m_{h}^{0}, p^{s}, e_{h}^{s}, e_{h}^{s m}, y^{s}\right) \in \mathbb{R}^{I} \times \mathbb{R} \times$ $\mathbb{R}_{++}^{C} \times \mathbb{R}_{++}^{C} \times \mathbb{R} \times \mathbb{R}^{I}$,

$$
\widehat{t^{s}}\left(b_{h}, m_{h}^{0}, p^{s}, e_{h}^{s}, e_{h}^{s m}, y^{s}\right) \leq\left(1-\varepsilon^{s}\right)\left(p^{s} e_{h}^{s}+e_{h}^{s m}+y^{s} b_{h}+m_{h}^{0}\right)
$$

2a. $\forall s \geq 1, \forall\left(b_{h}, m_{h}^{0}, p^{s}, e_{h}^{s}, e_{h}^{s m}, y^{s}\right)$,

$$
\widehat{t}^{s}\left(b_{h}, m_{h}^{0}, p^{s}, e_{h}^{s}, e_{h}^{s m}, y^{s}\right) \geq 0
$$

2b. $\forall s \geq 1 \quad \exists \eta^{s} \in(0,1)$ such that $\forall\left(b_{h}, m_{h}^{0}, p^{s}, e_{h}^{s}, e_{h}^{s m}, y^{s}\right)$,

$$
\sum_{h=1}^{H} \widehat{t}^{s}\left(b_{h}, m_{h}^{0}, p^{s}, e_{h}^{s}, e_{h}^{s m}, y^{s}\right) \geq \eta^{s} \sum_{h=1}^{H}\left(p^{s} e_{h}^{s}+e_{h}^{s m}+y^{s} b_{h}+m_{h}^{0}\right)
$$

3. $-\widehat{t}^{s}\left(p^{s}, e_{h}^{s}, e_{h}^{s m}, y^{s}, b_{h}, m_{h}^{0}\right)$ is $C^{2}$ with respect to $\left(b_{h}, m_{h}^{0}\right)$ and quasi-concave in $\left(b_{h}, m_{h}^{0}\right)$.

4a. $\forall s \geq 1, \exists \varepsilon^{s m} \in(0,1)$ such that $\forall\left(b_{h}, m_{h}^{0}, p^{s}, e_{h}^{s}, e_{h}^{s m}, y^{s}\right), \frac{\partial \widehat{t}^{s}}{\partial m_{h}^{0}}$ belongs to a compact subset of $\mathbb{R}$.

4b. $\forall s \geq 1, \forall i \in\{1, \ldots, I\}, \exists \varepsilon^{s i} \in(0,1)$ such that $\forall\left(b_{h}, m_{h}^{0}, p^{s}, e_{h}^{s}, e_{h}^{s m}, y^{s}\right)$, $\frac{\partial \widehat{t}^{s}}{\partial b_{h}^{i}}$ belongs to a compact subset of $\mathbb{R}$.

Observe that $p^{s} e_{h}^{s}+e_{h}^{s m}+y^{s} b_{h}+m_{h}^{0}$ is just the total wealth of household $h$ in state $s \geq 1$.

Assumption 6.1 is sufficient to insure that taxes are not so high to eat up some household's wealth in some state.

Assumption 6.2a requires in each state each household to be asked to pay non-negative taxes; $2 \mathrm{~b}$ that aggregate taxes are strictly positive - in fact, bounded away from zero.

Assumption 6.3 is a very mild one and basically requires the tax to be nonregressive in money and asset return. A convex function of the form $\widehat{\widehat{t}}: \mathbb{R}_{++} \rightarrow$ $\mathbb{R}, \widehat{\hat{t}}\left(p^{s} e_{h}^{s}+e_{h}^{s m}+y^{s} b_{h}+m_{h}^{0}\right)$ does satisfy Assumption 6.3.

Assumption 6.4 is satisfied if the marginal tax rates for money and asset demand belong to $[0,1] .^{12}$

Definition $7 E \equiv\left\{e^{m} \in \mathbb{R}^{(S+1) H}:\right.$ Assumption 5 is satisfied $\}$;

$$
\mathcal{T} \equiv\{\widehat{t}: \text { Assumption } 6 \text { is satisfied }\} \text {. }
$$

\footnotetext{
${ }^{12}$ Assumption 6.3 is used to show sufficiency of Kuhn-Tucker conditions. The other conditions are mainly used in Lemma 19.
} 
A relevant example of the function $\widehat{t}^{s}$ which satisfies the above assumptions is presented below.

For each state $s \geq 1$, define

$$
\begin{gathered}
T^{s} \equiv\left\{t^{s}=\left(t_{k}^{s}\right)_{k=0}^{K^{s}} \in \mathbb{R}^{K^{s}+1}: 0<t_{0}^{s}<t_{1}^{s}<\ldots<t_{K^{s}}^{s}\right\} \\
\bar{W}^{s} \equiv\left\{\bar{w}^{s} \equiv\left(\bar{w}_{k}^{s}\right)_{k=0}^{K^{s}} \in \mathbb{R}^{K^{s}+1}: \bar{w}_{0}^{s} \equiv 0<\bar{w}_{1}^{s}<\ldots<\bar{w}_{K^{s}}^{s}\right\}
\end{gathered}
$$

$t \equiv\left(t^{s}\right)_{s=1}^{S}$; moreover, $T \equiv \times_{s=1}^{S} T^{s}, \bar{w} \equiv\left(\bar{w}^{s}\right)_{s=1}^{S}, \bar{W} \equiv \times_{s=1}^{S} \bar{W}^{s}$. Call tax class $k$ in state $s$ the interval $\left(\bar{w}_{k}^{s}, \bar{w}_{k+1}^{s}\right]$ for $k \in\left\{0,1, \ldots, K^{s}-1\right\}$ and the interval $\left(\bar{w}_{K}^{s},+\infty\right)$ for $k=K^{s}$. We can then define the following tax function

$$
\begin{aligned}
& \widehat{t}^{s}: \mathbb{R}_{++}^{C} \times \mathbb{R}_{++}^{C} \times T^{s} \times \bar{W}^{s} \rightarrow \mathbb{R} \\
& \widehat{t}^{s}:\left(p^{s}, e_{h}^{s}, t^{s}, \bar{w}^{s}\right) \mapsto\left\{\begin{array}{lll}
t_{0}^{s} p^{s} e_{h}^{s} & \text { if } & p^{s} e_{h}^{s} \in\left(0, \bar{w}_{1}^{s}\right] \\
\sum_{k^{\prime}=0}^{k-1} t_{k^{\prime}}^{s}\left(\bar{w}_{k^{\prime}+1}^{s}-\bar{w}_{k^{\prime}}^{s}\right)+t_{k}^{s}\left(p^{s} e_{h}^{s}-\bar{w}_{k}^{s}\right) & \text { if } & p^{s} e_{h}^{s} \in\left(\bar{w}_{k}^{s}, \bar{w}_{k+1}^{s}\right] \\
\sum_{k^{\prime}=0}^{K^{s}-1} t_{1}^{s}\left(\bar{w}_{k^{\prime}+1}^{s}-\bar{w}_{k^{\prime}}^{s}\right)+t_{K^{s}}^{s}\left(p^{s} e_{h}^{s}-\bar{w}_{K^{s}}^{s}\right) & \text { if } & p^{s} e_{h}^{s} \in\left(\bar{w}_{K^{s}}^{s},+\infty\right]
\end{array}\right.
\end{aligned}
$$

An economy is completely described by an element $\mathcal{E} \equiv\left(e, e^{m}, \widehat{t}, Y, u\right) \in$ $\mathbb{R}_{++}^{G H} \times \mathcal{T} \times \mathcal{M} \times \mathcal{U}$

Household $h$ 's maximization problem is the following one. For given $\mathcal{E}$, $p \in \mathbb{R}_{++}^{G}$ and $q \in \mathbb{R}^{I}$

$$
\begin{aligned}
& \max _{\left(x_{h}, b_{h}, m_{h}^{0}\right) \in \mathbb{R}_{++}^{G} \times \mathbb{R}^{I} \times \mathbb{R}} \quad u_{h}\left(x_{h}\right) \\
& \text { s.t. } \\
& -p^{0}\left(x_{h}^{0}-e_{h}^{0}\right)-m_{h}^{0}+e_{h}^{0 m}-q b_{h}=0 \\
& -p^{s}\left(x_{h}^{s}-e_{h}^{s}\right)+m_{h}^{0}+e_{h}^{s m}+y^{s} b_{h}-\widehat{t}^{s}(\ldots)=0 \quad \text { for } s \geq 1 \\
& m_{h}^{0} \geq 0
\end{aligned}
$$

Observe that for each $h$ and $s \geq 1, m_{h}^{s}=\widehat{t}^{s}(\ldots)$. Moreover, $(x, b, m)$ satisfies market clearing conditions if

$$
\begin{aligned}
& \sum_{h=1}^{H}\left(x_{h}^{\backslash}-e_{h}^{\backslash}\right)=0 \\
& \sum_{h=1}^{H=1} b_{h}=0 \\
& \sum_{h=1}^{H}\left(m_{h}^{0}-e_{h}^{0 m}\right)=0 \\
& \sum_{h=1}^{H}\left[\left(m_{h}^{0}+e_{h}^{s, m}\right)-\widehat{t}^{s}(\ldots)\right]=0 \quad s \geq 1
\end{aligned}
$$

where $x_{h}^{\backslash} \equiv\left(x_{h}^{s c}\right)_{c \neq 1} \in \mathbb{R}_{++}^{G-(S+1)}$ and $e_{h}^{\backslash} \equiv\left(e_{h}^{s c}\right)_{c \neq 1} \in \mathbb{R}_{++}^{G-(S+1)}$. We can then give the following definition of equilibrium.

Definition $8\left(x, b, m^{0}, p, q\right) \in \mathbb{R}_{++}^{G H} \times \mathbb{R}^{I H} \times \mathbb{R}^{H} \times \mathbb{R}_{++}^{G} \times \mathbb{R}^{I}$ is a monetary equilibrium for $\mathcal{E}$ if:

1. for all $h=1, \ldots, H,\left(x_{h}, b_{h}, m_{h}^{0}\right)$ solves problem (2) at $(p, q, \mathcal{E})$; 
2. $\left(x, b, m^{0}\right)$ satisfies market clearing conditions (3) at $\mathcal{E}$.

For technical reasons, and without any loss of generality, we define an equilibrium using the prices of good 1 in each state $s$ as the unit of measure of values, i.e., we choose those goods as per state numeraire goods. Formally, we have what follows. Define

$$
\begin{aligned}
& \text { for each } s \geq 0, \widehat{p}^{s} \equiv \frac{p^{s}}{p^{s 1}} \in \mathbb{R}_{++}^{C} \text {, } \\
& \widehat{p}^{s \backslash} \equiv\left(\frac{p^{s c}}{p^{s 1}}\right)_{c \neq 1} \in \mathbb{R}_{++}^{C-1}, \\
& \widehat{p}^{\backslash} \equiv\left(p^{s \backslash}\right)_{s=0}^{S} \in \mathbb{R}_{++}^{G-(S+1)} \\
& \widehat{q} \equiv \frac{q}{p^{01}}
\end{aligned}
$$

and

$$
\begin{aligned}
& \text { for each } s \geq 0, q^{s m} \equiv \frac{1}{p^{s 1}} \in \mathbb{R}_{++}, \\
& q^{m} \equiv\left(q^{s m}\right)_{s=0}^{S} \in \mathbb{R}_{++}^{S++}
\end{aligned}
$$

Observe that

$$
p^{s}=\frac{\widehat{p}^{s}}{q^{s m}}
$$

The obvious interpretation of $q^{s m}$ is the price of money in state $s$ in terms of the numeraire good 1 in that state.

We can now give a definition of equilibrium which turns out to be equivalent to Definition 8 - see Lemma 10 below.

Definition $9\left(x, b, m^{0}, \widehat{p} \backslash, \widehat{q}, q^{m}\right) \in \mathbb{R}_{++}^{G H} \times \mathbb{R}^{I H} \times \mathbb{R}^{H} \times \mathbb{R}_{++}^{G-(S+1)} \times \mathbb{R}^{I} \times \mathbb{R}_{++}^{S+1}$ is a numeraire equilibrium for $\mathcal{E}$ if:

1. for all $h=1, \ldots, H,\left(x_{h}, b_{h}, m_{h}^{0}\right)$ solves problem

$$
\begin{aligned}
& \max _{\left(x_{h}, b_{h}, m_{h}^{0}\right) \in \mathbb{R}_{++}^{G} \times \mathbb{R}^{I} \times \mathbb{R}} \quad u_{h}\left(\widehat{x}_{h}\right) \\
& \text { s.t. } \\
& -\widehat{p}^{0}\left(x_{h}^{0}-e_{h}^{0}\right)+q^{0 m}\left(-m_{h}^{0}+e_{h}^{0 m}\right)-\widehat{q} b_{h}=0 \\
& -\widehat{p}^{s}\left(x_{h}^{s}-e_{h}^{s}\right)+q^{s m}\left(m_{h}^{0}+e_{h}^{s m}+y^{s} b_{h}\right)-q^{s m} \widehat{t}^{s}(\ldots)=0 \quad s \geq 1 \\
& m_{h}^{0} \geq 0 \\
& \text { at }\left(\widehat{p} \backslash, \widehat{q}, q^{m}, \mathcal{E}\right) ;
\end{aligned}
$$

2. $\left(x, b, m^{0}\right)$ satisfies market clearing conditions

$$
\begin{aligned}
& \sum_{h=1}^{H}\left(x_{h}^{\backslash}-e_{h}^{\backslash}\right)=0 \\
& \sum_{h=1}^{H} b_{h}=0 \\
& \sum_{h=1}^{H}\left(m_{h}^{0}-e_{h}^{0 m}\right)=0 \\
& \sum_{h=1}^{H}\left[\left(m_{h}^{0}+e_{h}^{s, m}\right)-\widehat{t}^{s}(\ldots)\right]=0 \quad s \geq 1
\end{aligned}
$$

It is then easy to show the following lemma. 
Lemma 10 If $\left(x, b, m^{0}, p, q\right)$ is a monetary equilibrium for $\mathcal{E}$, then

$$
\left(x, b, m^{0}, \widehat{p}=\left(\frac{p^{s}}{p^{s 1}}\right)_{s=0}^{S}, \widehat{q}=\frac{q}{p^{01}}, q^{m}=\left(\frac{1}{p^{s 1}}\right)_{s=0}^{S}\right)
$$

is a numeraire equilibrium for $\mathcal{E}$, and

if $\left(x, b, m^{0}, \widehat{p} \backslash, \widehat{q}, q^{m}\right)$ is a numeraire equilibrium for $\mathcal{E}$, then

$$
\left(x, b, m^{0}, p=\left(\frac{\widehat{p}^{s}}{q^{s m}}\right)_{s=0}^{S}, q=\frac{\widehat{q}}{q^{0 m}}\right)
$$

is a monetary equilibrium for $\mathcal{E}$.

The above lemma says nothing but monetary and numeraire equilibria differ only for the unit of measure of prices. In the former cases, in each spot, prices of goods and prices of assets are expressed in terms of units of money; in the latter, in each spot, prices of goods, prices of assets and prices of money are expressed in terms of units of good 1.

With innocuous abuse of notation, we take away the symbol "^ " from prices.

To get a more concise way of writing budget equations, define also ${ }^{13}$

$$
\Phi(p) \equiv\left(\begin{array}{cccc}
p^{0} & & & \\
& p^{1} & & \\
& & \ddots & \\
& & & p^{S}
\end{array}\right)_{(S+1) \times G}, \widetilde{\mathbf{1}} \equiv\left(\begin{array}{c}
-1 \\
\mathbf{1}
\end{array}\right)_{(S+1) \times 1}
$$

with $\mathbf{1} \equiv(1, \ldots, 1) \in \mathbb{R}^{S}$,

$$
q^{m \mathbf{1}} \equiv\left(q^{s m}\right)_{s=1}^{S} \text { and } \widetilde{q}^{m} \equiv\left(\begin{array}{c}
-q^{0 m} \\
q^{m \mathbf{1}}
\end{array}\right)_{(S+1) \times 1}
$$

Given an arbitrary vector $v \in \mathbb{R}^{n}, d g(v)$ is an $n$ diagonal matrix with $v$ as diagonal, and

$$
R_{m} \equiv\left[\begin{array}{c}
-q \\
d g\left(q^{m \mathbf{1}}\right) Y
\end{array}\right]_{(S+1) \times I} \equiv\left[\begin{array}{c}
-q \\
Y_{m}
\end{array}\right]
$$

Observe that

$$
\operatorname{rank}\left[R_{m} \mid \widetilde{q}^{m}\right]=I+1
$$

Define also

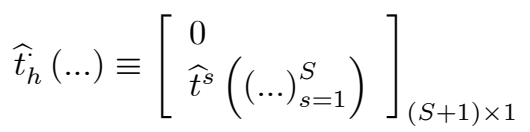

Then, the budget constraints in problem (4) can be written as

$$
-\Phi(p)\left(x_{h}-e_{h}\right)+d g\left(q^{m}\right)\left(m_{h}^{0} \widetilde{\mathbf{1}}+e_{h}^{m}-\widehat{t}_{h}(\ldots)\right)+R_{m} b_{h}=0
$$

\footnotetext{
${ }^{13}$ An empty space in a matrix denotes a zero submatrix.
} 


\section{Existence and Generic Regularity of Equilib- ria}

\subsection{Existence}

To show existence we take for fixed an arbitrary $(\widehat{t}, Y, u) \in \mathcal{T} \times \mathcal{M} \times \mathcal{U}$. The relevant exogenous variable space is then

$$
\Omega \equiv \mathbb{R}_{++}^{G H} \times E
$$

with generic element

$$
\omega \equiv\left(e, e^{m}\right)
$$

Define also the set of endogenous variables as

$$
\Xi \equiv\left(\mathbb{R}_{++}^{G} \times \mathbb{R}_{++}^{S+1} \times \mathbb{R}^{I} \times \mathbb{R} \times \mathbb{R}\right)^{H} \times \mathbb{R}_{++}^{G-(S+1)} \times \mathbb{R}^{I} \times \mathbb{R}_{++}^{S+1}
$$

with generic element

$$
\xi \equiv\left(\left(x_{h}, \lambda_{h}, b_{h}, m_{h}^{0}, \mu_{h}\right)_{h=1}^{H}, p^{\backslash}, q, q^{m}\right)
$$

We can now describe equilibria using the system of Kuhn-Tucker conditions - which characterize solutions to the households' maximization problems - and market clearing conditions (5). Define

$F: \Xi \times \Omega \rightarrow \mathbb{R}^{\operatorname{dim} \Xi}, F:(\xi, \omega) \mapsto$ left hand side of system (6) below

(h.1) $\quad D_{x_{h}} u_{h}\left(x_{h}\right)-\lambda_{h} \Phi(p)=0$

$(h .2) \quad-\Phi(p)\left(x_{h}-e_{h}\right)+d g\left(q^{m}\right)\left(\left(m_{h}^{0} \widetilde{\mathbf{1}}+e_{h}^{m}-t_{h}(\ldots)\right)\right)+R_{m} b_{h}=0$

(h.3) $\quad \lambda_{h} R\left(q, q^{m \mathbf{1}}, \widehat{t}\right)=0$

(h.4) $\quad \lambda_{h} \widetilde{q}^{m}(\widehat{t})+\mu_{h}=0$

(h.5) $\min \left\{\mu_{h}, m_{h}^{0}\right\}=0$

(M1) $\quad \sum_{h=1}^{H}\left(x_{h}^{\backslash}-e_{h}^{\backslash}\right)=0$

(M2) $\quad \sum_{h=1}^{H} b_{h}=0$

(M3) $\quad \sum_{h=1}^{H}\left(m_{h}^{0}-e_{h}^{0, m}\right)=0$

(M4) $\quad \sum_{h=1}^{H}\left[\left(m_{h}^{0}+e_{h}^{s, m}\right)-\widehat{t}^{s}(\ldots)\right]=0$

where budget constraints can be rewritten as follows

$$
\begin{aligned}
& -p^{0}\left(x_{h}^{0}-e_{h}^{0}\right)+q^{0 m}\left(-m_{h}^{0}+e_{h}^{0 m}\right)-q b_{h}=0 \\
& -p^{s}\left(x_{h}^{s}-e_{h}^{s}\right)+q^{s m}\left(m_{h}^{0}+e_{h}^{s m}+y^{s} b_{h}\right)-q^{s m} \widehat{t}^{s}(\ldots)=0 \quad s \geq 1
\end{aligned}
$$

Remark 11 From equation (M3) in system (6) and Assumption 5.2, in equilibrium there is $h$ such that $m_{h}^{0}>0$ and $\mu_{h}=0$. 

ria $^{14}$.

We now want to apply the following theorem to prove existence of equilib-

Theorem 12 Let $M$ and $N$ be two $C^{2}$ boundaryless manifolds of the same dimension, $y \in N$ and $F, G: M \rightarrow N$ be such that $F$ is $C^{0}$ and $G$ is $C^{0}$ and $C^{1}$ in an open neighborhood of $G^{-1}(y), y$ is a regular value for $G, \# G^{-1}(y)$ is odd, there exists a continuous homotopy $H$ from $F$ to $G$ such that $H^{-1}(y)$ is compact. Then $F^{-1}(y) \neq \emptyset$.

Definition $13 \xi \in \Xi$ is an extended equilibrium vector for $\omega \in \Omega$ if $F(\xi, \omega)=$ 0 .

With innocuous abuse of terminology, we will call $\xi$ simply an equilibrium. To verify the assumptions of Theorem 12, we proceed as follows. We first construct an appropriate "test economy" $\omega^{*}$ using a Pareto optimal allocation. Then we construct the needed homotopy $H$ - see (9) - defining the function $G \equiv F_{\omega^{*}}$. Finally, we show the other needed results, i.e., there exists $\xi^{*} \in \Xi$ such that $\xi^{*} \in G^{-1}(0)$ - Lemma $15,\left\{\xi^{*}\right\}=G^{-1}(0)$ - Lemma 16, $D_{\xi} G\left(\xi^{*}\right)$ has full rank - Lemma 18, $H^{-1}(0)$ is compact - Lemma 19.

Consider an arbitrary good endowment $e \in \mathbb{R}_{++}^{C H}$ and a Pareto optimal allocation $x^{*}$ feasible at $e$, i.e., such that $\sum_{h=1}^{H} x_{h}^{*}=\sum_{h=1}^{H} e_{h}$. It is well known that there exists a unique solution $\left(x^{*}, \theta_{\backslash 1}^{*} \equiv\left(\theta_{h}^{*}\right)_{h \neq 1}, \gamma^{*}\right) \in \mathbb{R}_{++}^{G H} \times \mathbb{R}_{++}^{H-1} \times \mathbb{R}_{++}^{G}$ to system

$$
\begin{array}{lll}
\text { (1) } & D u_{1}\left(x_{1}\right)-\gamma & =0 \\
(2) & \theta_{h} D u_{h}\left(x_{h}\right)-\gamma & =0 \\
(3) & \left(u_{h}\left(x_{h}\right)-u_{h}\left(x_{h}^{*}\right)\right)_{h \neq 1} & =0 \\
(4) & -\sum_{h=1}^{H} x_{h}+\sum_{h=1}^{H} x_{h}^{*} & =0
\end{array}
$$

Define $\theta_{1}^{*}=1$. Then the test economy $\omega^{*} \equiv\left(e^{*}, e^{m *}\right)$ is defined as follows.

$$
\begin{aligned}
e^{*} & \equiv x^{*} & \\
e_{h}^{0 m *} \equiv \frac{1}{4} \min _{s \geq 1}\left\{x_{h}^{s 1 *}\right\}>0 & & \text { for each } h \\
e_{h}^{s m *} \equiv \frac{1}{2} x_{h}^{s 1 *}-e_{h}^{0 m *} \geq \frac{1}{4} x_{h}^{s 1 *}>0 & & \text { for each } h \text { and } s \geq 1
\end{aligned}
$$

Define

$$
H_{\omega}: \Xi \times[0,1] \rightarrow \mathbb{R}^{d i m} \Xi, \quad(\xi, \tau) \mapsto \text { left hand side of system (9) below }
$$

\footnotetext{
${ }^{14}$ The theorem is a well-known result in degree thoery; for a proof, see, for example, Villanacci and others (2002), Chapter 7.
} 
(h.5) $\min \left\{\mu_{h}, m_{h}^{0}\right\}=0$

$$
\begin{aligned}
& D_{x_{h}} u_{h}\left(x_{h}\right)-\lambda_{h} \Phi(p)=0 \\
& -\Phi(p)\left(x_{h}-\left((1-\tau) e_{h}+\tau x_{h}^{*}\right)\right)+d g\left(q^{m}\right)\left[\left(m_{h}^{0} \widetilde{\mathbf{1}}+\left((1-\tau) e_{h}^{m}+\tau e_{h}^{m *}\right)+\right.\right. \\
& \left.-\left(0,\left((1-\tau) \widehat{t}^{s}(\ldots)+\tau\left(\frac{1}{2} \frac{1}{q^{s m}} x_{h}^{s 1 *}\right)\right)_{s=1}^{S}\right)\right]+R_{m} b_{h}=0 \\
& \lambda_{h}\left[d g\left(q^{m \mathbf{1}}\right)\left[y^{s i}-(1-\tau) \frac{\partial \widehat{t}^{s}}{\partial b_{h}^{i}}\right]_{s \geq 1, i}\right]=0 \\
& \lambda_{h}\left[d g\left(q^{m \mathbf{1}}\right)\left[\mathbf{1}-(1-\tau) \frac{\partial \widehat{t}^{s}}{\partial m_{h}^{0}}\right]_{s \geq 1}\right]+\mu_{h}=0 \\
& \min ^{0 m}\left\{\mu_{h}, m_{h}^{0}\right\}=0 \\
& \sum_{h=1}^{H}\left[x_{h}^{\backslash}-\left[(1-\tau) e_{h}^{\backslash}+\tau x_{h}^{\downarrow *}\right]\right]=0 \\
& \sum_{h=1}^{H} b_{h}=0 \\
& \sum_{h=1}^{H}\left[m_{h}^{0}-\left[(1-\tau) e_{h}^{0 m}+\tau e_{h}^{0 m *}\right]\right]=0 \\
& \sum_{h=1}^{H} m_{h}^{0}+\left[(1-\tau) e_{h}^{s m}+\tau e_{h}^{s m *}\right]-\sum_{h=1}^{H}\left((1-\tau) \widehat{t}^{s}(\ldots)+\tau\left(\frac{1}{2} \frac{1}{q^{s m}} x_{h}^{s 1 *}\right)\right)=0
\end{aligned}
$$

where budget constraints could be also be written as

$$
\begin{array}{ll}
-p^{0}\left(x_{h}^{0}-\left[(1-\tau) e_{h}^{0}+\tau x_{h}^{0 *}\right]\right)+q^{0 m}\left(-m_{h}^{0}+\left[(1-\tau) e_{h}^{0 m}+\tau e_{h}^{0 m *}\right]\right)-q b_{h}=0 & \\
-p^{s}\left(x_{h}^{s}-\left[(1-\tau) e_{h}^{s}+\tau x_{h}^{s *}\right]\right)+q^{s m}\left(m_{h}^{0}+\left[(1-\tau) e_{h}^{s m}+\tau e_{h}^{s m *}\right]+y^{s} b_{h}\right)+ & \\
-q^{s m}\left[(1-\tau) \widehat{t}^{s}(\ldots)+\tau\left(\frac{1}{2} \frac{1}{q^{s m}} x_{h}^{s 1 *}\right]=0\right. & s \geq 1
\end{array}
$$

Define also

$$
G: \Xi \rightarrow \mathbb{R}^{\operatorname{dim} \Xi}, \quad \xi \mapsto H_{\omega}(\xi, 1)
$$

Observe that

$$
H_{\omega}(\xi, 0)=F(\xi, \omega)=F_{\omega}(\xi)
$$

Remark 14 The basic idea used in constructing the homotopy is simple: it "links" the true economy to a fictitious economy via a convex combination. The fictitious economy is one in which endowments of goods and money are those defined in (8) and the tax function is $\frac{1}{2}$ of the value of the endowment of the numeraire good in each state $s \geq 1$.

Comparing system (7) and system $G(\xi)=0$, we get the following lemma. 
Lemma $15 \xi^{*} \equiv\left(x^{*}, \lambda^{*}, b^{*}, m^{0 *}, \mu^{*}, p^{{ }^{*}}, q^{*}, q^{m *}\right) \in G^{-1}(0)$, where

$$
\begin{array}{llll}
\lambda_{h}^{s *}=\frac{\gamma^{s 1 *}}{\theta_{h}^{*}} & & \text { for each } s \geq 0 \text { and for each } h \\
b_{h}^{*} & =0 & & \text { for each } h \\
m_{h}^{0 *} & =e_{h}^{0 m *} & & \text { for each } h \\
\mu_{h}^{*} & =0 & & \text { for each } h \\
p^{s \backslash *} & =\frac{\gamma^{s * 1}}{\gamma^{s 1 *}} & & \text { for each } s \geq 0 \\
q^{*} & =\sum_{s=1}^{S} \frac{\gamma^{s 1 *}}{\gamma^{01 *}} y^{s} & \\
q^{0 m *} & =\sum_{s=1}^{S} \frac{\gamma^{s 1 *}}{\gamma^{01 *}} & \\
q^{m \mathbf{1} *} & =\mathbf{1} &
\end{array}
$$

Lemma $16\left\{\xi^{*}\right\}=G^{-1}(0)$.

Proof. From the previous lemma, it is enough to show that if $G(\widehat{\xi})=0$, then $\widehat{\xi} \equiv\left(\widehat{x}, \widehat{\lambda}, \widehat{b}, \widehat{m}^{0}, \widehat{\mu}, \widehat{p} \backslash, \widehat{q}, \widehat{q}^{m}\right)=\xi^{*}$. In fact, $\widehat{x}=x^{*}$, otherwise Pareto optimality of $x^{*}$ would be contradicted. The equality between the other components of $\widehat{\xi}$ and of $\xi^{*}$ follows from $G\left(x^{*}, \widehat{\lambda}, \widehat{b}, \widehat{m}^{0}, \widehat{\mu}, \widehat{p} \backslash, \widehat{q}, \widehat{q}^{m}\right)=0$.

Remark 17 Since $m^{0 *}=e_{h}^{0 m *}>0=\mu_{h}^{*}$, in a small enough neighborhood of $G^{-1}(0)=\xi^{*}$

$$
\min \left\{\mu_{h}, m_{h}^{0}\right\}=\mu_{h}
$$

Therefore $G$ is continuous in such a neighborhood, and the corresponding assumption of Theorem 12 is satisfied.

Lemma $18 D_{\xi} G\left(\xi^{*}\right)$ has rank $\operatorname{dim} \Xi$.

Proof. We want to show that if $D_{\xi} G\left(\xi^{*}\right) \Delta \xi=0$, then $\Delta \xi=0$. Assuming otherwise, and using the differentiably strictly quasi-concavity of $u$, leads to a contradiction.

Lemma 19 For each $\omega \in \Omega, H_{\omega}^{-1}(0)$ is compact.

Proof. We have to show that all sequences $\left(\xi^{v}\right)_{v}$ in $H_{\omega}^{-1}(0)$, up to a subsequence, do converge to an element of $H_{\omega}^{-1}(0)$.

The convergence of $\left(x^{v}\right)_{v}$ follows from Assumption 1 and the fact $\left(x_{h}, b_{h}, m_{h}^{0}, \lambda_{h}, \mu_{h}\right)$ satisfies equations $(h .1)-(h .5)$ in system (9) if and only if $\left(x_{h}, b_{h}, m_{h}^{0}\right)$ solve the following problem.

$$
\begin{aligned}
& \max _{\left(x_{h}, b_{h}, m_{h}^{0}\right) \in \mathbb{R}_{++}^{G} \times \mathbb{R}^{I} \times \mathbb{R}} u_{h}\left(x_{h}\right) \\
& \text { s.t. } \\
& \text { (h.2) in system (9) } \\
& m_{h}^{0} \geq 0
\end{aligned}
$$

Below, we list the other components of $\xi^{v}$ and the main reasons for which they converge. 
$\mu_{\bar{h}}^{v}$ for some $\bar{h}$ : (M3) in (9) and Assumption 5;

$\lambda_{h}^{s v}:(h .1)$ in (9) and Assumption 1;

$p^{v}:(h .1)$ in $(9)$;

$q^{m v}$ : (M2), (M.3) and (M.4) in (9) and Assumptions 5.2 and 6.1 and 6.2;

$\left(\mu_{h}^{v}\right)_{h \neq \bar{h}}:(h .4)$ in (9) and 6.4.a.;

$q^{v}:(h .3)$ in (9) and 6.4.b.;

$\left(b^{v}, m^{0 v}\right):(h .2)$ in (9), Assumptions 4, 6.1 and 6.2.a.

Finally, since $\left\{\left(\xi^{v}, \tau^{v}\right)\right\} \subseteq H_{\omega}^{-1}(0)$ means that for every $v$

$$
H_{\omega}\left(\xi^{v}, \tau^{v}\right)=0
$$

taking limits of both sides and using the continuity of $H_{\omega}$, we get the desired result.

From the previous Lemmas, we finally get the desired existence theorem.

Theorem 20 For any $\omega \in \Omega$, there exists $\xi \in \Xi$ such that $F(\xi, \omega)=0$.

\subsection{Generic Regularity}

In this section we show that generically equilibria are finite and they locally depend from exogenous variables in a smooth manner if the tax function satisfies the following Assumption ${ }^{15}$ - where with innocuous abuse of notation we still use the symbol $\widehat{t}^{s}$.

Assumption 21 The tax function is continuous and takes the following form. For $s \geq 1$,

$$
\widehat{t}^{s}: \mathbb{R}_{++} \rightarrow \mathbb{R}, \widehat{t}^{s}: w^{s} \mapsto \widehat{t}^{s}\left(w^{s}\right)
$$

with

$$
w^{s}=\frac{p^{s}}{q^{s m}} e_{h}^{s}+e_{h}^{s m}+y^{s} b_{h}+m_{h}^{0}
$$

and it is such that

$$
\forall h, \forall s \geq 1, \forall w_{h}^{s}, D \widehat{t}^{s}\left(w_{h}^{s}\right) \in(0,1)
$$

The assumption simply says that each increment of wealth is non-trivially taxed and it cannot be completely taken away by the government.

Definition $22 \omega \in \Omega$ is a regular economy if

1. there exists $r \in \mathbb{N}$ such that $\left(F_{\omega}\right)^{-1}(0)=\left\{\xi^{i}\right\}_{i=1}^{r}$;

2. for each $i, D_{\xi} F_{\omega}\left(\xi^{i}\right)$ has full row rank;

3. for each $i$, there exists an open set $U \subseteq \Omega$, an open set $V_{i} \subseteq \mathbb{R}^{\operatorname{dim} \Xi}$ and a unique function $g_{i}: U \rightarrow V_{i}$ such that $\left(\xi^{i}, \omega\right) \in V_{i} \times \bar{U}, g_{i}$ is $C^{1}$, $g_{i}(\omega)=\xi^{i}$ and for every $\omega^{\prime} \in U, F\left(g_{i}\left(\omega^{\prime}\right), \omega^{\prime}\right)=0$.

\footnotetext{
${ }^{15}$ Using the strategy followed in this section, other forms of the tax function could be analyzed.
} 
Let $\mathcal{R}$ be the set of regular economies

Proposition $23 \mathcal{R}$ is an open and full measure subset of $\Omega$.

To show the above proposition, we need to preliminary show that $F$ is differentiable at least in a neighborhood of $F^{-1}(0)$. In fact, both the tax functions and the functions $\min \left\{m_{h}^{0}, \mu_{h}\right\}$ are not (necessarily) differentiable. To solve the problem related to the tax functions either we simply strengthen Assumption 21 , requiring $\widehat{t}^{s}$ to be differentiable, or for a more specific form of the function itself we have to show the following condition holds true. ${ }^{16}$

Condition 24 There exists an open and full measure subset $D^{*}$ of $\Omega$ such that $\forall s \geq 1, \forall \omega \in D^{*}$ and $\forall \xi \in \Xi$ such that $F(\xi, \omega)=0$ it is the case that $\widehat{t}^{s}=\widetilde{t}^{s}$ in a small enough neighborhood of $(\xi, \omega)$ and $\widetilde{t}^{s}$ is differentiable.

The main Lemma needed to prove Proposition 23 is presented below.

Lemma 25 For each $u \in \mathcal{U}$, there exists an open and full measure subset $D^{* *}$ of $\Omega$ such that $\forall \omega \in D^{* *}, \forall \xi$ such that $F(\xi, \omega)=0$ and $\forall h \in \mathcal{H}$, either $m_{h}^{0}>0$ or $\mu_{h}>0$.

Proofs of both Lemma 25 and Proposition 23 use the same strategy adopted in the corresponding results in next sections (see Lemma 26 and Proposition 29 ) and they are therefore omitted.

\section{Non-neutrality of Monetary Policy for the Piece- wise Linear Tax Function}

The possibility of showing effectiveness of planner intervention is prevented by the generality of the tax functions described in the previous sections. Several choices of a more specific function could be analyzed following the strategy presented below. For the sake of simplicity, we analyze the case of one of the most commonly used tax schedules: the piecewise linear function described in (1), i.e.,

$\widehat{t}^{s}\left(p^{s}, e_{h}^{s}, t^{s}, \bar{w}^{s}\right)=\max \left\{t_{0}^{s} p^{s} e_{h}^{s}, \sum_{k^{\prime}=0}^{k-1} t_{k^{\prime}}^{s}\left(\bar{w}_{k^{\prime}+1}^{s}-\bar{w}_{k^{\prime}}^{s}\right)+t_{k}^{s}\left(p^{s} e_{h}^{s}-\bar{w}_{k}^{s}\right)\right.$ for $\left.k \in\left\{1, \ldots, K^{s}\right\}\right\}$

Just to make notation sufficiently readable we take $K=2$ (and we set, for $\left.s \geq 1, \bar{w}^{s} \equiv \bar{w}_{1}^{s}\right)$. In subsection 4.1, we check that Condition 24 holds; in subsection 4.2, we prove the Pareto improving result.

${ }^{16}$ That latter strategy is used in Section 4.1. 


\subsection{Generic Regularity}

The (numeraire) equilibrium system is the following one.

$$
\begin{aligned}
& D_{x_{h}} u_{h}\left(x_{h}\right)-\lambda_{h} \Phi(p)=0 \\
& -p^{0}\left(x_{h}^{0}-e_{h}^{0}\right)-q^{s m}\left(m_{h}^{0}-e_{h}^{0 m}\right)-q b_{h}=0 \\
& -p^{s}\left(x_{h}^{s}-e_{h}^{s}\right)+q^{s m}\left(m_{h}^{0}+e_{h}^{s m}+y^{s} b_{h}\right)-\max \left\{t_{0}^{s} p^{s} e_{h}^{s}, q^{s m}\left(t_{0}^{s}-t_{1}^{s}\right) \bar{w}^{s}+t_{1}^{s} p^{s} e_{h}^{s}\right\}=0 \quad s \geq 1 \\
& \lambda_{h} R_{m}=0 \\
& \lambda_{h} \widetilde{q}^{m}+\mu_{h}=0 \\
& \min \left\{\mu_{h}, m_{h}^{0}\right\}=0 \\
& \sum_{h=1}^{H}\left(x_{h}^{\backslash}-e_{h}^{\backslash}\right)=0 \\
& \sum_{h=1}^{H} b_{h}=0 \\
& \sum_{h=1}^{H=1}\left(m_{h}^{0}-e_{h}^{0, m}\right)=0 \\
& \sum_{h=1}^{H} q^{s m}\left(m_{h}^{0}+e_{h}^{s, m}\right)-\sum_{h=1}^{H} \max \left\{t_{0}^{s} p^{s} e_{h}^{s}, q^{s m}\left(t_{0}^{s}-t_{1}^{s}\right) \bar{w}^{s}+t_{1}^{s} p^{s} e_{h}^{s}\right\}=0
\end{aligned}
$$

As in the previous section, we want to show the set of regular economies, as defined there, is an open and full measure subset of $\Omega$. Therefore, we have to preliminary show the following statement.

Generically in the space $\Omega, 1$. households are not border line cases with respect to the demand of money (i.e., it is not the case that $m_{h}^{0}=0$ and $\left.\mu_{h}=0\right)$, and 2. they are not at a kink of the tax function.

The main idea to get the above result is to introduce fictitious models whose collections of equilibrium sets contain the equilibria we are investigating. This idea is formalized in Cass, Siconolfi and Villanacci (2001). That contribution deals with a model in which only border line cases with respect to the demand of money can arise. In the case of piecewise linear tax functions that idea simply specializes in what follows: for each state $s \geq 1$, consider as exogenously given the sets of households which are in each tax class. To formalize the above idea we need some definitions and notation.

Consider the set $\mathcal{P}_{2}(\mathcal{H})$ of all partitions of $\mathcal{H}$ into two subsets. For each element $\left(\left\{\mathcal{H}_{0}^{s}, \mathcal{H}_{1}^{s}\right\}\right)_{s=1}^{S} \in\left(\mathcal{P}_{2}(\mathcal{H})\right)^{S}$, we can define a fictitious model imposing that, in each state $s$, irrespectively of each household $h$ ' wealth, she pays taxes in that state according to the first (respectively, second) component of the tax function if $h \in \mathcal{H}_{0}^{s}$ (respectively, $\mathcal{H}_{1}^{s}$ ).

More precisely $\forall s \geq 1$

$$
\text { if } h \in \mathcal{H}_{0}^{s} \text {, then } \widehat{t}^{s}\left(p^{s}, e_{h}^{s}, t^{s}, \bar{w}^{s}\right)=t_{0}^{s} p^{s} e_{h}^{s}
$$

and

$$
\text { if } h \in \mathcal{H}_{1}^{s} \text {, then } \widehat{t}^{s}\left(p^{s}, e_{h}^{s}, t^{s}, \bar{w}^{s}\right)=q^{s m}\left(t_{0}^{s}-t_{1}^{s}\right) \bar{w}^{s}+t_{1}^{s} p^{s} e_{h}^{s}
$$

We also define $H_{k}^{s} \equiv \# \mathcal{H}_{k}^{s}$, for $k \in\{0,1\}$ and $s \geq 1$.

Defined

$$
\widetilde{e}_{h}^{s m} \equiv\left\{\begin{array}{lll}
e_{h}^{s m} & \text { if } & h \in \mathcal{H}_{0}^{s} \\
e_{h}^{s m}+\left(t_{1}^{s}-t_{0}^{s}\right) \bar{w}^{s} & \text { if } & h \in \mathcal{H}_{1}^{s}
\end{array}\right.
$$


the equilibrium system associated to (the fictitious model) $\left(\left\{\mathcal{H}_{0}^{s}, \mathcal{H}_{1}^{s}\right\}\right)_{s=1}^{S}$ can be written as follows.

$$
\begin{array}{ll}
D_{x_{h}} u_{h}\left(x_{h}\right)-\lambda_{h} \Phi(p)=0 & \\
-p^{0}\left(x_{h}^{0}-e_{h}^{0}\right)-q^{0 m}\left(m_{h}^{0}-e_{h}^{0 m}\right)-q b_{h}=0 & s \geq 1, \text { if } h \in \mathcal{H}_{0}^{s} \\
-p^{s}\left(x_{h}^{s}-e_{h}^{s}\right)+q^{s m}\left(m_{h}^{0}+\widetilde{e}_{h}^{s m}+y^{s} b_{h}\right)-t_{0}^{s} p^{s} e_{h}^{s}=0 & s \geq 1, \text { if } h \in \mathcal{H}_{1}^{s} \\
-p^{s}\left(x_{h}^{s}-e_{h}^{s}\right)+q^{s m}\left(m_{h}^{0}+\widetilde{e}_{h}^{s m}+y^{s} b_{h}\right)-t_{1}^{s} p^{s} e_{h}^{s}=0 & \\
\lambda_{h} R_{m}=0 & \\
\lambda_{h} \widetilde{q}^{m}+\mu_{h}=0 & \\
\min \left\{\mu_{h}, m_{h}^{0}\right\}=0 & \\
\sum_{h=1}^{H}\left(x_{h}^{\backslash}-e_{h}^{\backslash}\right)=0 & \\
\sum_{h=1}^{H} b_{h}=0 & \\
\sum_{h=1}^{H}\left(m_{h}^{0}-e_{h}^{0, m}\right)=0 & s \geq 1 \\
\quad q^{s m}\left(\sum_{h_{0} \in \mathcal{H}_{0}^{s}}\left(m_{h_{0}}^{0}+e_{h_{0}}^{s m}\right)+\sum_{h_{1} \in \mathcal{H}_{1}^{s}}\left(m_{h_{1}}^{0}+e_{h_{1}}^{s m}+\left(t_{1}^{s}-t_{0}^{s}\right) \bar{w}^{s}\right)\right)+ & \\
\quad-\left(\sum_{h_{0} \in \mathcal{H}_{0}^{s}} t_{0}^{s} p^{s} e_{h_{0}}^{s}+\sum_{h \in \mathcal{H}_{1}^{s}} t_{1}^{s} p^{s} e_{h_{1}}^{s}\right)=0 &
\end{array}
$$

It is also convenient to rewrite the equilibrium system as follows. Consider the set

$$
J \equiv\{0,1\}^{S}
$$

with generic term $j$, and $\mathcal{P}_{J}(\mathcal{H})$ as the set of all partitions of $\mathcal{H}$, where each partition has cardinality $\# J=2^{S}$. For each element $\left\{\mathcal{H}_{j}\right\}_{j \in J} \in \mathcal{P}_{J}(\mathcal{H})$ we can define a fictitious model imposing that

if $h \in \mathcal{H}_{j}, s \geq 1$, and the $s$-th component of $j$ is equal to $k$, then $h$ pays taxes

$$
\begin{cases}t_{0}^{s} p^{s} e_{h}^{s} & \text { if } k=0 \\ q^{s m}\left(t_{0}^{s}-t_{1}^{s}\right) \bar{w}^{s}+t_{1}^{s} p^{s} e_{h}^{s} & \text { if } k=1\end{cases}
$$

Let $h_{j}$ be a generic element of $\mathcal{H}_{j}$ and define $t_{j} \equiv\left(t_{j}^{s}\right)_{s=1}^{S}$ where

$$
t_{j}^{s} \equiv\left\{\begin{array}{lll}
t_{0}^{s} & \text { if } & s \text {-th component of } j \text { is } 0 \\
t_{1}^{s} & \text { if } & s \text {-th component of } j \text { is } 1
\end{array}\right.
$$

and

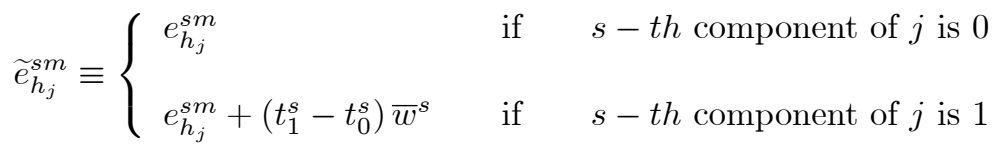

Then the equilibrium system associated to (the fictitious model) $\left\{\mathcal{H}_{j}\right\}_{j \in J}$ can be written as follows. 


$$
\begin{array}{ll}
\left(h_{j} .1\right) & D_{x_{h_{j}}} u_{h_{j}}\left(x_{h_{j}}\right)-\lambda_{h_{j}} \Phi(p)=0 \\
& \quad-p^{0}\left(x_{h_{j}}^{0}-e_{h_{j}}^{0}\right)-q^{0 m}\left(m_{h_{j}}^{0}-e_{h_{j}}^{0 m}\right)-q b_{h_{j}}=0 \\
\left(h_{j} .2\right) & \quad-p^{s}\left(x_{h_{j}}^{s}-e_{h_{j}}^{s}\right)+q^{s m}\left(m_{h_{j}}^{0}+\widetilde{e}_{h_{j}}^{s m}+y^{s} b_{h_{j}}\right)-t_{j}^{s} p^{s} e_{h_{j}}^{s}=0 \quad s \geq 1 \\
& \left.\quad h_{j} .3\right) \\
\left(h_{h_{j}} R_{m}=0\right. \\
\left(h_{j} .4\right) & \lambda_{h_{j}} \widetilde{q}^{m}+\mu_{h_{j}}=0 \\
& \min \left\{\mu_{h_{j}}, m_{h_{j}}^{0}\right\}=0 \\
(M 1) & \sum_{h_{j}=1}^{H}\left(x_{h_{j}}^{\backslash}-e_{h_{j}}^{\backslash}\right)=0 \\
(M 2) & \sum_{h_{j}=1}^{H} b_{h_{j}}=0 \\
(M 3) & \sum_{h_{j}=1}^{H}\left(m_{h_{j}}^{0}-e_{h_{j}}^{0, m}\right)=0 \\
(M 4) & \sum_{j \in J} \sum_{h \in \mathcal{H}_{j}} q^{s m}\left(m_{h}^{0}+\widetilde{e}_{h}^{s m}\right)-\sum_{j \in J} \sum_{h \in \mathcal{H}_{j}} t_{j}^{s} p^{s} e_{h}^{s}=0
\end{array}
$$

To simplify notation, denote by $i$ a generic element of $\mathcal{P}_{J}(\mathcal{H})$ and by $F^{i}(\xi, \omega)$ the left hand side of system (11) above.

We now repeat the above strategy to deal with border line cases with respect to the demand of money. Let $\mathcal{P}$ be the family of all partitions of $\mathcal{H}$ into three subsets $\mathcal{H}_{1}, \mathcal{H}_{2}$ and $\mathcal{H}_{3}$, with $\mathcal{H}_{3} \neq \emptyset$. In the equilibrium system (10), in place of $\min \left\{m_{h}^{0}, \mu_{h}\right\}=0$ substitute $\mu_{h}=0$ for $h \in \mathcal{H}_{1}, m_{h}^{0}=0$ for $h \in \mathcal{H}_{2}$, and $m_{h}^{0}=0$ and $\mu_{h}=0$ for $h \in \mathcal{H}_{3}$.

Define

$$
F_{\mathcal{H}_{1}, \mathcal{H}_{2}, \mathcal{H}_{3}}^{i}: \Xi \times \Omega \rightarrow \mathbb{R}^{\operatorname{dim} \Xi+\# \mathcal{H}_{3}}
$$

as the function which associates the left hand side of the fictitious $i$ equilibrium system modified as explained above.

Observe that given an arbitrary $(\xi, \omega) \in F^{-1}(0)$, we can define endogenously $i(\xi, \omega)$ and $\mathcal{H}_{1}(\xi, \omega), \mathcal{H}_{2}(\xi, \omega), \mathcal{H}_{2}(\xi, \omega)$ in the obvious way. Then

$$
(\xi, \omega) \in\left(F_{\mathcal{H}_{1}(\xi, \omega), \mathcal{H}_{2}(\xi, \omega), \mathcal{H}_{2}(\xi, \omega)}^{i(\xi)}\right)^{-1}(0)
$$

All the above discussion allows us to conclude that

$$
\text { each } F_{\mathcal{H}_{1}, \mathcal{H}_{2}, \mathcal{H}_{3}}^{i} \text { is differentiable on all its domain }
$$

and

$$
F^{-1}(0) \subseteq \cup_{i \in \mathcal{P}_{J}(\mathcal{H}),\left\{\mathcal{H}_{1} \mathcal{H}_{2} \mathcal{H}_{3}\right\} \in \mathcal{P}}\left(F_{\mathcal{H}_{1}, \mathcal{H}_{2}, \mathcal{H}_{3}}^{i}\right)^{-1}(0)
$$

and also $\forall \omega \in \Omega,\left(F_{\omega}\right)^{-1}(0) \subseteq \cup_{i \in \mathcal{P}_{J}(\mathcal{H}),\left\{\mathcal{H}_{1} \mathcal{H}_{2} \mathcal{H}_{3}\right\} \in \mathcal{P}}\left(F_{\mathcal{H}_{1}, \mathcal{H}_{2}, \mathcal{H}_{3} \mid \omega}\right)^{-1}(0)$.

We now present the strategy of the proof that border line cases and kinks of the tax function are "rare".

Border line cases.

Define the sets

$$
B_{\mathcal{H}_{1}, \mathcal{H}_{2}, \mathcal{H}_{3}}^{i} \equiv\left\{\omega \in \Omega: \exists \xi \text { such that } F_{\mathcal{H}_{1}, \mathcal{H}_{2}, \mathcal{H}_{3}}^{i}(\xi, \omega)=0\right\}
$$




$$
\begin{aligned}
C \equiv\left\{(\xi, \omega) \in F^{-1}(0)\right. & \left.: \exists h \text { such that } m_{h}^{0}=\mu_{h}=0\right\} \\
D^{*} & \equiv \Omega \backslash p r(C)
\end{aligned}
$$

By definition of $D^{*}, \forall \omega \in D^{*}$ and $\forall \xi$ such that $F(\xi, \omega)=0$,

$$
\forall h \in \mathcal{H} \text {, either } m_{h}^{0}>0 \text { or } \mu_{h}>0
$$

Define also

$$
B^{m} \equiv \cup_{i \in \mathcal{P}_{J}(\mathcal{H}),\left\{\mathcal{H}_{1} \mathcal{H}_{2} \mathcal{H}_{3}\right\} \in \mathcal{P}} B_{\mathcal{H}_{1} \mathcal{H}_{2} \mathcal{H}_{3}}^{i}
$$

By definition of $B^{m}$, each economy $\omega$ for which an equilibrium is such that some household is at a border line in contained in $B^{m}$.

Then ${ }^{17}$

$$
D^{*} \supseteq \Omega \backslash B^{m}
$$

Observe that since $B_{\mathcal{H}_{1}, \mathcal{H}_{2}, \mathcal{H}_{3}}$ is of measure zero, $\underset{\left\{\mathcal{H}_{1}, \mathcal{H}_{2}, \mathcal{H}_{3}\right\} \in \mathcal{P}}{\cup} B_{\mathcal{H}_{1}, \mathcal{H}_{2}, \mathcal{H}_{3}}^{i}$ is of measure zero as well. Observe also the for any $\left\{\mathcal{H}_{1}, \mathcal{H}_{2}, \mathcal{H}_{3}\right\} \in \mathcal{P}$ and $\forall h_{3} \in \mathcal{H}_{3}$, we have that $B_{\mathcal{H}_{1}, \mathcal{H}_{2}, \mathcal{H}_{3}}^{i} \subseteq B_{\mathcal{H}_{1}, \mathcal{H}_{2},\left\{h_{3}\right\}}^{i}$. Therefore, it is enough to show that

$$
B_{\mathcal{H}_{1}, \mathcal{H}_{2},\left\{h_{3}\right\}}^{i} \text { has measure zero }
$$

Kinks of the tax function.

Define $F^{i *} \equiv F_{\mid D^{*}}^{i}$. Consider the family $\mathcal{F}$ of all subsets of $\mathcal{H}$. Define

$$
\widetilde{\mathcal{F}} \equiv\left\{\left\{\mathcal{H}^{s}\right\}_{s=1}^{S} \in \mathcal{F}^{S}: \exists s \text { such that } \mathcal{H}^{s} \neq \emptyset\right\}
$$

Define $F_{\left\{\mathcal{H}^{s}\right\}_{s=1}^{S}}^{i *}$ as the equilibrium function in the fictitious model $i$ with the following appended equations

$$
\forall s, \forall h \in \mathcal{H}^{s}, \quad p^{s} e_{h}^{s}-\bar{w}^{s}
$$

For each $\left\{\mathcal{H}^{s}\right\}_{s=1}^{S} \in \widetilde{\mathcal{F}}$, define

$$
B_{\left\{\mathcal{H}^{s}\right\}_{s=1}^{S}}^{i} \equiv\left\{\omega \in \Omega: \exists \xi \in \Xi \text { such that } F_{\left\{\mathcal{H}^{s}\right\}_{s=1}^{S}}^{i *}(\xi, \omega)=0\right\}
$$

Observe that $B_{\left\{\mathcal{H}^{s}\right\}_{s=1}^{S}}^{i}$ is the set of equilibria of fictitious model $i$ where, for each $s \geq 1$, households in $\mathcal{H}^{s}$ are at a kink of the "true" tax function in state $s$.

Define

$$
C^{\prime} \equiv\left\{(\xi, \omega) \in F^{-1}(0): \exists h, s \geq 1 \text { such that } p^{s} e_{h}^{s}-\bar{w}^{s}=0\right\}
$$

and

$$
D^{* *} \equiv D^{*} \backslash \operatorname{pr}\left(C^{\prime}\right)
$$

\footnotetext{
${ }^{17}$ We cannot use the equality sign in (14), because $B_{\mathcal{H}_{1}, \mathcal{H}_{2}, \mathcal{H}_{3}}$ contains economies $\omega$ such that $F_{\mathcal{H}_{1}, \mathcal{H}_{2}, \mathcal{H}_{3}}(\xi, \omega)=0$ and for which some components $m_{h}^{0}$ and $\mu_{h}$ of $\xi$ may be negative, and some househods pay a tax which is not that one of the tax class they belong to.
} 
By definition of $D^{* *}, \forall \omega \in D^{* *}$ and $\forall \xi$ such that $F(\xi, \omega)=0$,

$$
\begin{aligned}
& \forall h, \in \mathcal{H}, s \geq 1 p^{s} e_{h}^{s}-\bar{w}^{s} \neq 0 \quad \text { and } \\
& \forall h \in \mathcal{H}, \text { either } m_{h}^{0}>0 \text { or } \mu_{h}>0
\end{aligned}
$$

Define

$$
B^{t} \equiv \cup_{i \in \mathcal{P}_{J}(\mathcal{H}),\left\{\mathcal{H}^{s}\right\}_{s=1}^{S} \in \mathcal{F}^{S}} B_{\left\{\mathcal{H}^{s}\right\}_{s=1}^{S}}^{i}
$$

Using the standard approach we can show that the sets $B_{\left\{\mathcal{H}^{s}\right\}_{s=1}^{S}}^{i}$, and therefore $B^{t}$, are closed and null. Then

$$
D^{* *} \supseteq D^{*} \backslash B^{t}
$$

Finally observe that for any $i \in \mathcal{P}_{J}(\mathcal{H})$, for any $\left\{\mathcal{H}^{s}\right\}_{s=1}^{S} \in \widetilde{\mathcal{F}}$, for any $s \geq 1$ such that $\mathcal{H}^{s} \neq \emptyset$ and for any $h^{s} \in \mathcal{H}^{s}$,

$$
B_{\left\{\mathcal{H}^{s}\right\}_{s=1}^{S}}^{i} \subseteq B_{\left\{\mathcal{H}^{s^{\prime} *}\right\}_{s^{\prime}=1}^{S}}^{i}
$$

where

$$
\mathcal{H}^{s^{\prime} *} \equiv\left\{\begin{array}{ccc}
\emptyset & \text { if } & s^{\prime} \neq s \\
\left\{h^{s}\right\} & \text { if } & s^{\prime}=s
\end{array}\right.
$$

Therefore, it is enough to show that

$$
B_{\left\{\mathcal{H}^{s^{\prime} *}\right\}_{s^{\prime}=1}^{S}}^{i} \text { has measure zero }
$$

Lemmas 26 and 27 below show that both conditions (15) and (17) hold. Both of them deal with the Jacobian of $F_{\mathcal{H}_{1}, \mathcal{H}_{2}, \mathcal{H}_{3}}^{i}$. The needed notation is presented in Appendix "Notation for the Jacobian of the equilibrium function".

Lemma $26 B_{\mathcal{H}_{1}, \mathcal{H}_{2},\left\{h_{3}\right\}}^{i}$ has measure zero.

Proof. That $B_{\mathcal{H}_{1}, \mathcal{H}_{2},\left\{h_{3}\right\}}$ is of measure zero follows from the parametric transversality theorem - see, for example, Hirsch (1976), Theorem 2.7, page 79 - and from the fact that zero is a regular value for $F_{\mathcal{H}_{1}, \mathcal{H}_{2},\left\{h_{3}\right\}}^{i}$. The basic idea of the proof is to use (the column of the derivative of $F_{\mathcal{H}_{1}, \mathcal{H}_{2},\left\{h_{3}\right\}}^{i}$ with respect to $e_{h}^{s m}$, multiplied by $\frac{1}{q^{s m}}$ ) to partially "clean" the column of the derivative with respect to $e_{h}^{s 1}$. Then the proof is similar to the analogous proof in Cass, Siconolfi and Villanacci (2001).

Lemma $27 B_{\left\{\mathcal{H}^{s^{\prime} *}\right\}_{s^{\prime}=1}^{S}}^{i}$ has measure zero.

Proof. To fix ideas take $s=1$ and $h=h_{j_{1}}$. Using the rank result in the previous lemma, it is enough to show that the partial Jacobian of the left hand sides of the following equations in system (11)

$$
\left(h_{j_{1}} \cdot 2\right),\left(h_{j_{2}} .2\right),(M 1),(M 3),(M .4)
$$


and of the appended component

$$
p^{1} e_{h_{j_{1}}}^{1}-\bar{w}^{1}
$$

with respect to the following variables

$$
\left(e_{h_{j_{1}}}, e_{h_{j_{1}}}^{m}, e_{h_{j_{2}}}^{\cdot}, e_{h_{j_{2}}}^{\backslash} e_{h_{j_{2}}}^{m}\right)
$$

has full row rank. That matrix is displayed below.

\begin{tabular}{|l|l|l|l|l|}
\hline$d g\left(\mathbf{1}-t_{j_{1}}\right)$ & $d g\left(q^{m}\right)$ & & & \\
\hline & & $d g\left(\mathbf{1}-t_{j_{2}}\right)$ & $\Psi_{p h_{j_{2}}}^{\backslash}$ & $d g\left(q^{m}\right)$ \\
\hline & & & $-I$ & \\
\hline & -10 & & & -10 \\
\hline$-d g\left(t_{j_{1}}\right)$ & $0 d g\left(q^{\mathbf{1 m}}\right)$ & $-d g\left(t_{j_{2}}\right)$ & $\psi_{0 h_{j_{2}}}$ & $0 d g\left(q^{\mathbf{1 m}}\right)$ \\
\hline 010 & & & & \\
\hline
\end{tabular}

and it can be shown to have full rank through elementary columns operations.

We need also the following Lemma.

Lemma $28 p r: F^{-1}(0) \rightarrow \Omega,(\xi, \omega) \mapsto \omega$ is proper.

Proof. The proof follows the same steps as Lemma 19.

The definition of regular economies presented in the previous section applies to the model with piecewise linear functions. Define $\mathcal{R}^{\prime}$ that set. We want then to prove the following Proposition.

Proposition $29 \mathcal{R}^{\prime}$ is an open and full measure subset of $\Omega$.

Proof. The result follows from the Transversality theorem and the fact that the image of a closed set via a proper function is closed. Besides existence theorem 20, the proof uses Lemmas 26, 27 and 28, and the construction described in this section which leads to conditions 12 and 13.

Remark 30 Consider a partition of any $\mathcal{H}_{j}$ into two arbitrary subsets $\mathcal{H}_{1 j}$ and $\mathcal{H}_{2 j}$. For our purposes (proof of other generic properties and possibility of Pareto improvements as defined in next section), the above proposition allows to make statements about the (non-differentiable) function $F$ using the family of functions

$$
\widehat{F}_{\left\{\mathcal{H}_{1 j}, \mathcal{H}_{2 j}\right\}_{j \in J}}: \Xi \times \mathcal{R}^{\prime} \times T \times \bar{W} \times \mathcal{U} \rightarrow \mathbb{R}^{\operatorname{dim} \Xi}
$$

where $\widehat{F}_{\left\{\mathcal{H}_{1 j}, \mathcal{H}_{2 j}\right\}_{j \in J}}(\xi, \omega, t, \bar{w}, u)$ is the left hand side of system (11), with equation $\left(h_{j} .5\right)$ substituted by $\mu_{h}=0$ if $h \in \mathcal{H}_{1 j}$ and $m_{h}^{0}=0$ if $h \in \mathcal{H}_{2 j}$.

With some innocuous abuse of notation, we call still $\widehat{F}$ the generic element of the above equilibrium functions. 
The following lemma is needed in the proof of (Case 2 in) Theorem 32.

Lemma 31 There exists an open and dense subset $D^{*}$ of $\mathcal{R}^{\prime} \times T \times \bar{W} \times \mathcal{U}$ such that $\forall(\omega, t, \bar{w}, u) \in D_{1}^{*}$ and $\xi$ such that $\widehat{F}(\xi, \omega, t, \bar{w}, u)=0$, it is the case that $\gamma_{1} \neq 0$, where $\gamma_{1}$ is

$$
\begin{aligned}
& 1-\sum_{s} \frac{q^{s m}}{q^{0 m}} \frac{1}{\sum_{h_{0} \in \mathcal{H}_{0}} t_{0}^{s} h_{h_{0}}^{s 2}+\sum_{h_{1} \in \mathcal{H}_{1}} t_{1}^{s} e_{h_{1}}^{s 2}} . \\
& \cdot\left(\sum_{h_{0} \in \mathcal{H}_{0}} \frac{\lambda_{h_{0}}^{s}}{\lambda_{h_{0}}^{0}}\left(x_{h_{0}}^{s 2}-e_{h_{0}}^{s 2}+t_{0}^{s} e_{h_{0}}^{s 2}\right)+\sum_{h_{1} \in \mathcal{H}_{1}} \frac{\lambda_{h_{1}}^{s}}{\lambda_{h_{1}}^{0}}\left(x_{h_{1}}^{s 2}-e_{h_{1}}^{s 2}+t_{1}^{s} e_{h_{1}}^{s 2}\right)\right)
\end{aligned}
$$

Proof. In the proof, since we take derivatives both with respect to $t_{0}^{s}$ and $t_{1}^{s}$, we obviously assume that $\mathcal{H}_{0}^{s} \neq \emptyset$ and $\mathcal{H}_{1}^{s} \neq \emptyset$. If that is not the case, the proof follows the same strategy, and it is explained in detail in del Mercato and Villanacci (2003).

The openness results follows from the properness of the projection from the equilibrium manifold to the parameter space. The density result follows from a repeated application of the transversality theorem to the function $\widehat{F}$ with some extra component appended to it. For $l \in\{1, \ldots, 4\}$, in Step $l$ below, we show that zero is a regular value for $\left(\widehat{F}, \gamma_{l}\right)$. A needed condition to show that is that the derivative of $\gamma_{l}$ with respect to a well chosen component of $(\xi, \omega, t)$ is different from zero. Therefore in Step $l+1$ we show that zero is a regular value for $\left(\widehat{F}, \gamma_{l+1}\right)$ where if $\gamma_{l+1} \neq 0$, then that derivative is different from zero

Step 1. There exists an open and dense subset $D_{1}^{*}$ of $\mathcal{R}^{\prime} \times T$ such that $\forall(\omega, t) \in D_{1}^{* *}$ and $\xi$ such that $\widehat{F}(\xi, \omega, t, \bar{w}, u)=0, \gamma_{1} \neq 0$.

The relevant Jacobian is obtained taking derivatives of the left hand sides of the following equations in system (11)

$$
\left(h_{j_{1}} .2\right),\left(h_{j_{2}} .2\right),(M 1),(M 3),(M .4)
$$

and of the appended component

$$
\gamma_{1}
$$

\begin{tabular}{|c|c|c|c|c|c|c|c|}
\hline$d g\left(\mathbf{1}-t_{j_{1}}\right)$ & $\Psi_{p h_{j_{1}}}^{\backslash}$ & $d g\left(q^{m}\right)$ & & & & $\stackrel{0}{0} v_{h_{j}}^{0}$ & $\stackrel{0}{0} v_{h_{j}}^{1}$ \\
\hline & & & $d g\left(\mathbf{1}-t_{j_{2}}\right)$ & $\Psi_{p h_{j_{2}}}^{\backslash}$ & $d g\left(q^{m}\right)$ & $\stackrel{0}{0} v_{h_{j}}^{0}$ & $\stackrel{0}{0} v_{h_{j}}^{1}$ \\
\hline & $-I$ & & & $-I$ & & & \\
\hline & & -10 & & & -10 & & \\
\hline \multirow[t]{2}{*}{$-d g\left(t_{j_{1}}\right)$} & $\psi_{0 h_{j_{1}}}^{\backslash}$ & $0 d g\left(q^{1 m}\right)$ & $-d g t_{j_{2}}$ & $\psi_{0 h_{j_{2}}}^{\backslash}$ & $0 d g q^{\mathbf{1} m}$ & $N_{t^{0}}$ & $N_{t^{1}}$ \\
\hline & $*$ & & & $*$ & & $*$ & $\gamma_{2}$ \\
\hline
\end{tabular}

with respect to the following variables

$$
\left(e_{h_{j_{1}}}, e_{h_{j_{2}}}^{\backslash}, e_{h_{j_{1}}}^{m}, e_{h_{j_{2}}}, e_{h_{j_{2}}}^{\backslash}, e_{h_{j_{2}}}^{m}, t_{0}, t_{1}\right)
$$

and it displayed below. ${ }^{18}$

${ }^{18} \mathrm{~A} *$ entry in a matrix denotes a non-zero submatrix. 
Using the supercolumn of $\left(e_{h}^{s m}\right)_{s=1}^{S}$ to erase the supercolumn of $t_{1}$, as explained in Remark 33 in the Appendix, we get

\begin{tabular}{|l|l|l|l|l|l|l|l|}
\hline$d g\left(\mathbf{1}-t_{j_{1}}\right)$ & $\Psi_{p h_{j_{1}}}^{\backslash}$ & $d g\left(q^{m}\right)$ & & & & $\begin{array}{c}0 \\
d g v_{h_{j}}^{0}\end{array}$ & \\
\hline & & & $d g\left(\mathbf{1}-t_{j_{2}}\right)$ & $\Psi_{p h_{j_{2}}}^{\backslash}$ & $d g\left(q^{m}\right)$ & $\begin{array}{c}0 \\
d g v_{h_{j}}^{0}\end{array}$ & \\
\hline & $-I$ & & & $-I$ & & & \\
\hline & & -10 & & & -10 & & \\
\hline$-d g\left(t_{j_{1}}\right)$ & $\psi_{0 h_{j_{1}}}^{\backslash}$ & $0 d g\left(q^{\mathbf{1 m}}\right)$ & $-d g t_{j_{2}}$ & $\psi_{0 h_{j_{2}}}^{\backslash}$ & $0 d g q^{\mathbf{1 m}}$ & $N_{t^{0}}$ & \\
\hline & $*$ & & & $*$ & & $*$ & $\gamma_{2}$ \\
\hline
\end{tabular}

and then $t_{1}$ can be used to perturb the last equation, as long as $\gamma_{2} \neq 0$, as shown below.

Step 2. There exists an open and dense subset $D_{2}^{*}$ of $D_{1}^{*}$ such that $\forall(\omega, t) \in$ $D_{2}^{*}$ and $\xi$ such that $\widehat{F}(\xi, \omega, t)=0, \gamma_{2} \neq 0$.

$\gamma_{2} \equiv \frac{\partial \gamma_{1}}{\partial t_{1}^{s}} \neq 0$ if the following quantity is different from zero:

$$
\begin{aligned}
& \left(\sum_{h_{1} \in \mathcal{H}_{1}} \frac{\lambda_{h_{1}}^{s}}{\lambda_{h_{1}}^{o}} e_{h_{1}}^{s 2}\right)\left(\sum_{h_{0} \in \mathcal{H}_{0}} t_{0}^{s} e_{h_{0}}^{s 2}+\sum_{h_{1} \in \mathcal{H}_{1}} t_{1}^{s} e_{h_{1}}^{s 2}\right)+ \\
& -\left(\sum_{h_{1} \in \mathcal{H}_{1}} e_{h_{1}}^{s 2}\right)\left(\sum_{h_{0} \in \mathcal{H}_{0}} \frac{\lambda_{h_{0}}^{s}}{\lambda_{h_{0}}^{0}}\left(x_{h_{0}}^{s 2}-e_{h_{0}}^{s 2}+t_{0}^{s} e_{h_{0}}^{s 2}\right)+\sum_{h_{1} \in \mathcal{H}_{1}} \frac{\lambda_{h_{1}}^{s}}{\lambda_{h_{1}}^{o}}\left(x_{h_{1}}^{s 2}-e_{h_{1}}^{s 2}+t_{1}^{s} e_{h_{1}}^{s 2}\right)\right)
\end{aligned}
$$

The relevant Jacobian is the following one.

\begin{tabular}{|l|l|l|l|l|l|l|c||}
\hline$d g\left(\mathbf{1}-t_{j_{1}}\right)$ & $\Psi_{p h_{j_{1}}}^{\backslash}$ & $d g\left(q^{m}\right)$ & & & & $\begin{array}{c}0 \\
d g v_{h_{j}}^{0}\end{array}$ & $\begin{array}{c}0 \\
d g v_{h_{j}}^{1}\end{array}$ \\
\hline & & & $d g\left(\mathbf{1}-t_{j_{2}}\right)$ & $\Psi_{p h_{j_{2}}}^{\backslash}$ & $d g\left(q^{m}\right)$ & $\begin{array}{c}0 \\
d g v_{h_{j}}^{0}\end{array}$ & $\begin{array}{c}0 \\
d g v_{h_{j}}^{1}\end{array}$ \\
\hline & $-I$ & & & $-I$ & & & \\
\hline & & -10 & & & -10 & & \\
\hline$-d g\left(t_{j_{1}}\right)$ & $\psi_{0 h_{j_{1}}}^{\backslash}$ & $0 d g\left(q^{\mathbf{1 m}}\right)$ & $-d g t_{j_{2}}$ & $\psi_{0 h_{j_{2}}}^{\backslash}$ & $0 d g\left(q^{\mathbf{1 m}}\right)$ & $N_{t^{0}}$ & $N_{t^{1}}$ \\
\hline & $*$ & & & $*$ & & $\gamma_{3}$ & \\
\hline
\end{tabular}

Again from Remark 33, we get

\begin{tabular}{|l|l|l|l|l|l|l|c||}
\hline$d g\left(\mathbf{1}-t_{j_{1}}\right)$ & $\Psi_{p h_{j_{1}}}^{\backslash}$ & $d g\left(q^{m}\right)$ & & & & & $\begin{array}{c}0 \\
d g v_{h_{j}}^{1}\end{array}$ \\
\hline & & & $d g\left(\mathbf{1}-t_{j_{2}}\right)$ & $\Psi_{p h_{j_{2}}}^{\backslash}$ & $d g\left(q^{m}\right)$ & & $\begin{array}{c}0 \\
d g v_{h_{j}}^{1}\end{array}$ \\
\hline & $-I$ & & & $-I$ & & & \\
\hline & & -10 & & & -10 & & \\
\hline$-d g\left(t_{j_{1}}\right)$ & $\psi_{0 h_{j_{1}}}^{\backslash}$ & $0 d g\left(q^{\mathbf{1 m}}\right)$ & $-d g t_{j_{2}}$ & $\psi_{0 h_{j_{2}}}^{\backslash}$ & $0 d g\left(q^{\mathbf{1 m}}\right)$ & & $N_{t^{1}}$ \\
\hline & $*$ & & & $*$ & & $\gamma_{3}$ & \\
\hline
\end{tabular}

We can then use $t_{0}^{s}$ to perturb the last row, as long as $\gamma_{3} \neq 0$, as shown below. 
To be able to prove the above statement, we have now to introduce a linear local parameterization of the utility function of each household. For any $u_{h} \in \mathcal{U}$ and for any household $h$, consider a $\alpha_{h} \equiv\left(\left(\alpha_{h}^{s c}\right)_{c=1}^{C}\right)_{s=0}^{S} \in \mathbb{R}^{G}$. For any $h$,define

$$
\begin{aligned}
& \widetilde{u}_{h}\left(., \alpha_{h}\right): \mathbb{R}_{++}^{G} \rightarrow \mathbb{R} \\
& \widetilde{u}_{h}\left(x_{h}, \alpha_{h}\right) \equiv \bar{u}_{h}\left(x_{h}\right)+\frac{1}{2} \beta_{h}\left(x_{h}\right) \alpha_{h} x_{h}
\end{aligned}
$$

where $\beta_{h}$ is a bump function centered around an equilibrium allocation (see Citanna, Kajii and Villanacci (1998), for details). For each $h$, there exists an open neighborhood $\mathcal{A}_{h}^{0}$ of $0 \in \mathbb{R}^{G}$ such that for each $\alpha_{h} \in \mathcal{A}_{h}^{0}$ we have that $\widetilde{u}_{h}\left(., A_{h}\right) \in \mathcal{U}_{h}$.

Step 3. There exists an open and dense subset $D_{3}^{*}$ of $D_{2}^{*} \times \mathcal{U}$ such that $\forall(\omega, u) \in D_{3}^{*}$ and $\xi$ such that $\widehat{F}(\xi, \omega, u)=0, \gamma_{3} \neq 0$.

$\gamma_{3} \equiv \frac{\partial \gamma_{2}}{\partial t^{0}} \neq 0$ if the following quantity is different from zero.

$$
\left(\sum_{h_{1} \in \mathcal{H}_{1}} \frac{\lambda_{h_{1}}^{s}}{\lambda_{h_{1}}^{0}} e_{h_{1}}^{s 2}\right)\left(\sum_{h_{0} \in \mathcal{H}_{0}} e_{h_{0}}^{s 2}\right)-\left(\sum_{h_{1} \in \mathcal{H}_{1}} e_{h_{1}}^{s 2}\right)\left(\sum_{h_{0} \in \mathcal{H}_{0}} \frac{\lambda_{h_{0}}^{s}}{\lambda_{h_{0}}^{0}} e_{h_{0}}^{s 2}\right)
$$

Using the proposed local finite parameterization of the utility function, the result is straightforward.

Step 4. There exists an open and dense subset $D_{4}^{*}$ of $D_{3}^{*} \times \mathcal{U}$ such that $\forall(\omega, u) \in D_{4}^{*}$ and and $\xi$ such that $\widehat{F}(\xi, \omega, u)=$, it is the case that $\gamma_{4} \neq 0$, where

$$
\gamma_{4} \equiv \frac{\partial \gamma_{3}}{\partial e_{h_{1}}^{s 2}}=\frac{\lambda_{h_{1}}^{s}}{\lambda_{h_{1}}^{0}}\left(\sum_{h_{0} \in \mathcal{H}_{0}} e_{h_{0}}^{s 2}\right)-\left(\sum_{h_{0} \in \mathcal{H}_{0}} \frac{\lambda_{h_{0}}^{s}}{\lambda_{h_{0}}^{0}} e_{h_{0}}^{s 2}\right)=\sum_{h_{0} \in \mathcal{H}_{0}}\left(\frac{\lambda_{h_{1}}^{s}}{\lambda_{h_{1}}^{0}}-\frac{\lambda_{h_{0}}^{s}}{\lambda_{h_{0}}^{0}}\right) e_{h_{0}}^{s 2}
$$

The proof is similar to that one of Step 2 , and requires that $\forall s, s^{\prime} \geq 1, s \neq s^{\prime}$ and $\forall h, h^{\prime}, h \neq h^{\prime}$

$$
\gamma_{5} \equiv \frac{\lambda_{h}^{s}}{\lambda_{h}^{s^{\prime}}}-\frac{\lambda_{h^{\prime}}^{s}}{\lambda_{h^{\prime}}^{s^{\prime}}} \neq 0
$$

i.e., the standard condition of Pareto inefficiency, which can be easily proved in our framework.

\subsection{Pareto Improvements}

To prove the main result of this section, we use the following general methodology described, for example, in Citanna, Kajii and Villanacci (1998) and in Chapter 15 in Villanacci and others (2002).

1. Starting from the equilibrium function $F(\xi, \omega, t, \bar{w}, u)$, we define a new equilibrium function $F_{1}(\xi, \omega, t, \bar{w}, u)$, taking into account the planner's intervention effects on agents behaviors via some policy tools $\rho \in \mathbb{R}^{T}$, and a function $F_{2}(\xi, \omega, t, \bar{w}, u)$, describing the constraints on the planner intervention. We then consider a function $\widetilde{F} \equiv\left(F_{1}, F_{2}\right)$ whose zeros are 
equilibria with planner's intervention. We can partition the vector $\tau$ of tools into two subvectors $\tau_{1} \in \mathbb{R}^{T-T_{2}}$ and $\tau_{2} \in \mathbb{R}^{T_{2}}$, where $\tau_{1}$ can be interpreted as the vector of independent tools and $\tau_{2}$ as the vector of dependent tools.

In our chosen type of intervention there are no constraints on planner intervention and no dependent tools.

2. We observe that there is a value $\bar{\tau}$ at which equilibria with and without planner's intervention coincide.

3. We define the function $U(\xi, \omega, t, \bar{w}, u)=u(x)$, and we analyze the local effect of a change in $\tau$ around $\bar{\tau}$ on $U$ when its arguments assume their equilibrium (with planner intervention) values.

To accomplish the analysis described in 3., we proceed through the following technical steps.

(a) As a consequence of Proposition 29 and, also keeping in mind the needed Lemma 31, we know that $\forall(\omega, t, \bar{w}, u) \in D^{*}$ there exists a neighborhood $N$ of $\bar{\tau}$ and a unique $C^{1}$ function $h(\tau)$ defined on $N$, such that for $\tau \in N$

$$
\widetilde{F}(\xi(\tau), \tau, \omega, t, \bar{w}, u)=0
$$

The function $h$ describes how equilibrium variables and dependent tools adjust to changes in planner's tools $\tau$. Then the function

$$
g_{(\omega, t, \bar{w}, u)}: N \rightarrow \mathbb{R}^{H}, \tau \mapsto U(\xi(\tau), \tau, \omega, t, \bar{w}, u)
$$

describes how the goal function changes when the planner uses her policy tools $\tau$ and variables move in the equilibrium set defined by $\widetilde{F}$.

(b) The goal of the analysis is to show that there exists an open and dense subset $S^{*} \subseteq \Omega \times T \times \bar{W} \times \mathcal{U}$ such that for each $(\omega, t, \bar{w}, u) \in S^{*}$, the planner can "move" the equilibrium value of the goal function in any directions locally around $g_{(\omega, t, \bar{w}, u)}(\bar{\tau})$, the value of the goal function in the case of no intervention. More formally, we need to show that $g_{(\omega, t, \bar{w}, u)}$ is essentially surjective at $\bar{\tau}$, i.e., the image of each open neighborhood of $\bar{\tau}$ contains an open neighborhood of $g_{(\omega, t, \bar{w}, u)}(\bar{\tau})$ in $\mathbb{R}^{H}$.

(c) A sufficient condition for $g_{(\omega, t, \bar{w}, u)}$ to satisfy the above described condition is that

$$
\operatorname{rank}\left[D_{\tau} g_{(\omega, t, \bar{w}, u)}(\bar{\tau})\right]_{H \times H}=H
$$

(d) The above statement holds in an open and dense subset $S^{*}$ of $\Omega \times$ $T \times \bar{W} \times \mathcal{U}$ if for each $(\omega, t, \bar{w}, u) \in S^{*}$ the following system has no 
solutions $(\xi, c) \in \Xi \times \mathbb{R}^{\operatorname{dim} \Xi+H}$ :

$$
\begin{cases}F(\xi, \omega, t, \bar{w}, u) & =0 \\ c^{T}\left[D_{(\xi, \tau)}(\widetilde{F}, G)(\xi, \bar{\tau}, \omega, t, \bar{w}, u)\right] & =0 \\ c^{T} c-1 & =0\end{cases}
$$

i. Openness of $S^{*}$. It follows from the properness of the projection.

ii. Density of $S^{*}$. We redefine the functions $F, \widetilde{F}, G$ replacing $\mathcal{U}$ in their domain with a open ball $\widehat{\mathcal{A}}$ in a finite Euclidean space with generic element $a \equiv\left(a_{h}\right)_{h=1}^{H}$, consistently with the local finite parametrization of the utility functions described in Citanna, Kajii and Villanacci $(1998)^{19}$. Call $\widehat{F}_{A}, \widetilde{F}_{A}, G_{A}$ the functions so obtained and define $F_{A}^{*}(\xi, \omega, t, \bar{w}, a)=0$ as

$$
\begin{cases}F_{A}(\xi, \omega, t, \bar{w}, a) & =0 \\ c^{T}\left[D_{(\xi, \tau)}\left(\widetilde{F}_{A}, G_{A}\right)(\xi, \bar{\tau}, \omega, t, \bar{w}, a)\right] & =0 \\ c^{T} c-1 & =0\end{cases}
$$

We are then left with showing that 0 is a regular value for $F_{A}^{*}$, i.e., either

A.

$F_{A}^{*}(\xi, \omega, t, \bar{w}, a)=0$ has no solutions $(\xi, c)$

for all values of $(\omega, t, \bar{w}, a)$, or in an open and dense subset of $\Omega \times T \times \bar{W} \times \widehat{\mathcal{A}}$

or

B. for each $(\xi, \omega, t, \bar{w}, a) \in\left(F_{A}^{*}\right)^{-1}(0)$, the Jacobian matrix

$$
D F_{A}^{*}((\xi, \omega, t, \bar{w}, a))=\left[\begin{array}{lll}
D_{\xi} F_{A}(\xi, \omega, t, \bar{w}, a) & \\
* & {\left[D_{(\xi, \tau)}\left(\widetilde{F}, G_{A}\right)(\ldots)\right]^{T} N(c)}
\end{array}\right]
$$

has full row rank. Since from generic regularity, $D_{\xi} F_{A}$ has full row rank in an open and dense subset of the parameter space, in order to show the above condition, it is enough to show that

$$
\begin{aligned}
& \text { for each }(\xi, \omega, t, \bar{w}, a) \in\left(F_{A}^{*}\right)^{-1}(0), \\
& {\left[\begin{array}{ll}
{\left[D_{(\xi, \tau)}\left(\widetilde{F}, G_{A}\right)(\ldots)\right]^{T}} & N(c) \\
c &
\end{array}\right]}
\end{aligned}
$$

has full rank

\footnotetext{
${ }^{19}$ Take $\left(\bar{u}_{h}\right)_{h=1}^{H} \in \mathcal{U}$. Let $\mathcal{A}_{h}$ be the set of $G \times G$ symmetric matrices. Take $A_{h} \in \mathcal{A}_{h}$. Then the used finite parameterization is

$$
\begin{aligned}
& \widetilde{u}_{h}\left(., A_{h}\right): \mathbb{R}_{++}^{G} \rightarrow \mathbb{R} \\
& \widetilde{u}_{h}\left(x_{h}, A_{h}\right) \equiv \bar{u}_{h}\left(x_{h}\right)+\frac{1}{2} \beta_{h}\left(x_{h}\right) \sum_{k}\left[\left(x_{h}-\bar{x}_{h}^{k}\right) A_{h}\left(x_{h}-\bar{x}_{h}^{k}\right)\right]
\end{aligned}
$$

where $\beta_{h}$ is a $C^{\infty}$ bump function centered around each equilibrium.
} 
We can now present the planner intervention.

Our choice of policy tools is simply a subsidy or tax $\rho_{h}$ of money on household $h$ for each household in period zero. Then $\rho \equiv\left(\rho_{h}\right)_{h=1}^{H} \in \mathbb{R}^{H}$ and, in fact, $\rho_{h}$ is a subsidy if positive and a tax if negative.

Observe that since the production of money is costless, there is no requirement on the total amount of subsidy. Therefore, we do not require the total amount of subsidies $\sum_{h=1}^{H} \rho_{h}$ to be equal to zero, as in the case of reallocation of a given good among households, and therefore no further intervention is needed in period 1.

The equilibrium system taking into consideration the planner intervention is the following one.

$$
\begin{array}{ll}
\left(h_{j} .1\right) & D_{x_{h_{j}}} u_{h_{j}}\left(x_{h_{j}}\right)-\lambda_{h_{j}} \Phi(p)=0 \\
& \quad-p^{0}\left(x_{h_{j}}^{0}-e_{h_{j}}^{0}\right)-q^{0 m}\left(m_{h_{j}}^{0}-e_{h_{j}}^{0 m}-\rho_{h}\right)-q b_{h_{j}}=0 \\
\left(h_{j} .2\right) & \quad-p^{s}\left(x_{h_{j}}^{s}-e_{h_{j}}^{s}\right)+q^{s m}\left(m_{h_{j}}^{0}+\widetilde{e}_{h_{j}}^{s m}+y^{s} b_{h_{j}}\right)-t_{j}^{s} p^{s} e_{h_{j}}^{s}=0 \quad s \geq 1 \\
& \quad h_{h_{j}} R_{m}=0 \\
\left(h_{j} .3\right) & \lambda_{h_{j}} \widetilde{q}^{m}+\mu_{h_{j}}=0 \\
\left(h_{j} .4\right) & \left.h_{j} .5\right) \\
(M 1) & \left.\sum_{h_{h_{j}}}, m_{h_{j}}^{0}\right\}=0 \\
(M 2) & \sum_{h_{j}=1}^{H}\left(x_{h_{j}}^{\backslash}-e_{h_{j}}^{\backslash}\right)=0 \\
(M 3) & \sum_{h_{j}=1}^{H} b_{h_{j}}=0 \\
(M 4) & \sum_{h_{j}=1}^{H}\left(m_{h_{j}}^{0}-e_{h_{j}}^{0 m}-\rho_{h}\right)=0 \\
& \sum_{j \in J} \sum_{h \in \mathcal{H}_{j}} q^{s m}\left(m_{h}^{0}+\widetilde{e}_{h}^{s m}\right)-\sum_{j \in J} \sum_{h \in \mathcal{H}_{j}} t_{j}^{s} p^{s} e_{h}^{s}=0
\end{array}
$$

Define

$$
\widetilde{F}: \Xi \times \mathbb{R}^{H} \times \Omega \times \mathcal{U} \rightarrow \mathbb{R}^{\operatorname{dim} \Xi}, \quad \widetilde{F}:(\xi, \rho, \omega, u) \mapsto(\text { Left Hand Side of 24) }
$$

We are now ready to state the main result of this section.

Theorem 32 For an open and dense subset $S^{*}$ of the set of the economies $(\omega, t, \bar{w}, u)$, at any equilibrium $\xi^{\prime}$, the function $g$ is essentially surjective at 0 . That is, there exists a vector of money subsidies (or taxes) which allows to Pareto improve (or impair) upon the equilibrium $\xi^{\prime}$.

The proof of the theorem consists in showing that either condition (22) or (23) hold consistently with different values of $c$ and it is presented in the Appendix "Proof of Theorem 32".

\section{Appendices}

\subsection{Notation for the Jacobian of the Equilibrium Function}

In what follows, we introduce the matrix notation needed to write the Jacobian of the equilibrium function. We group notation in terms of the variables we differentiate with respect to. 
Prices of goods

$$
\begin{aligned}
& \hat{\Lambda}_{h}=\left[\begin{array}{ccc}
0_{C-1} & \cdots & \cdots \\
-\lambda_{h}^{0} I_{c-1} & \cdots & \\
\cdots & \cdots & 0_{C-1} \\
\cdots & & -\lambda_{h}^{0} I_{C-1}
\end{array}\right]_{G \times[G-(S+1)]} \\
& Z_{h_{j}}^{\backslash} \equiv\left[\begin{array}{ccc}
-\left(x_{h_{j}}^{0 \backslash}-e_{h_{j}}^{0 \backslash}\right) & -\left(x_{h_{j}}^{1 \backslash}-e_{h_{j}}^{1 \backslash}\right)-t_{j}^{1} e_{h_{j}}^{1 \backslash} & \\
& & -\left(x_{h_{j}}^{S \backslash}-e_{h_{j}}^{1 \backslash}\right)-t_{j}^{S} e_{h_{j}}^{S \backslash}
\end{array}\right] \equiv \\
& \equiv\left[\begin{array}{ccc}
-z_{h_{j}}^{0 \backslash} & & \\
& -\widetilde{z}_{h_{j}}^{\backslash \backslash} & \\
& & -\widetilde{z}_{h_{j}}^{S \backslash}
\end{array}\right]_{(S+1) \times[G-(S+1)]} \equiv\left[\begin{array}{cc}
-z_{h_{j}}^{0 \backslash} & \\
& \widetilde{Z}_{h_{j}}^{1 \backslash}
\end{array}\right] \\
& \begin{array}{l}
{\left[0 \mid E^{\backslash}\right] \equiv} \\
\equiv\left[\begin{array}{cccc}
0_{C-1} & -\left(\sum_{h_{0} \in \mathcal{H}_{0}^{1}} t_{0}^{1} e_{h_{0}}^{1 \backslash}+\sum_{h_{1} \in \mathcal{H}_{1}^{1}} t_{1}^{1} e_{h_{1}}^{1 \backslash}\right) & & \\
& & \ddots & \\
& & & -\left(\sum_{h_{0} \in \mathcal{H}_{0}^{S}} t_{0}^{S} e_{h_{0}}^{S \backslash}+\sum_{h_{1} \in \mathcal{H}_{1}^{S}} t_{1}^{S} e_{h_{1}}^{S \backslash}\right)
\end{array}\right] \equiv \\
\equiv\left[\begin{array}{llll}
0_{C-1} & -\left(\sigma^{1 c}\right)_{c \neq 1} & & \\
& & \ddots & \\
& & -\left(\sigma^{S c}\right)_{c \neq 1}
\end{array}\right]_{S \times[G-(S+1)]}
\end{array}
\end{aligned}
$$

Prices of money

$$
\begin{gathered}
Y_{\lambda_{h}^{1}} \equiv\left[\left(\operatorname{diag} \lambda_{h}^{\mathbf{1}}\right) Y\right]_{S \times I}=\left[\begin{array}{cc}
\lambda_{h}^{1} y^{11} & \lambda_{h}^{1} y^{1 I} \\
\lambda_{h}^{S} y^{S 1} & \lambda_{h}^{S} y^{S I}
\end{array}\right] \\
D \equiv \operatorname{diag}\left(\sum_{h=1}^{H}\left(m_{h}^{0}+\widetilde{e}_{h_{j}}^{s m}\right)\right)_{s=1}^{S}=\operatorname{diag}\left(\sum_{j \in J} \sum_{h \in \mathcal{H}_{j}} t_{j}^{s} p^{s} e_{h}^{s}\right)_{s=1}^{S} \\
\widetilde{M}_{h^{j}} \equiv\left[\begin{array}{c}
\left(-m_{h^{j}}^{0}+e_{h^{j}}^{0 m}\right) \\
\equiv\left[\begin{array}{c}
\widetilde{m}_{h^{j}}^{0} \\
\operatorname{diag}\left(\widetilde{m}_{h^{j}}^{s}\right)_{s=1}^{S}
\end{array}\right]_{(S+1) \times(S+1)}\left(m_{h^{j}}^{0}+\widetilde{e}_{h^{j}}^{s m}+y^{s} b_{h^{j}}\right)_{s=1}^{S}
\end{array}\right] \equiv
\end{gathered}
$$


Endowments of goods

$$
\begin{aligned}
& \Psi_{p h_{j}}^{\backslash} \equiv\left[\begin{array}{llll}
p^{0 \backslash} & & & \\
& \left(1-t_{j}^{1}\right) p^{1} & & \\
& & \cdots & \\
& & & \left(1-t_{j}^{S}\right) p^{S \backslash}
\end{array}\right]_{(S+1) \times[G-(S+1)]} \\
& \Psi_{p h_{j}}^{\bullet} \equiv \operatorname{diag}\left(\mathbf{1}-t_{j}\right)
\end{aligned}
$$

where $t_{j} \equiv\left(t_{j}^{s}\right)_{s=0}^{S}$ and $t_{j}^{0} \equiv 0$.

$$
\begin{gathered}
\Psi_{0 h_{j}}^{\backslash} \equiv\left[\begin{array}{ccc}
-t_{j}^{1} p^{1 \backslash} & & \\
& \cdots & \\
& & -t_{j}^{S} p^{S \backslash}
\end{array}\right]_{S \times[S(C-1)]} \\
\Psi_{0 h_{j}} \equiv \operatorname{diag}\left(-t_{j}\right)
\end{gathered}
$$

Tax parameters

$$
\begin{aligned}
& v_{h_{j}}^{0} \equiv\left(v_{h_{j}}^{0 s}\right)_{s=1}^{S}, v_{h_{j}}^{1} \equiv\left(v_{h_{j}}^{1 s}\right)_{s=1}^{S} \text { and } \\
& v_{h_{j}}^{0 s} \equiv\left\{\begin{array}{lll}
-p^{s} e_{h_{j}}^{s} & \text { if } & s \text {-th component of } j \text { is } 0 \\
-q^{s m} \bar{w}^{s} & \text { if } & s \text {-th component of } j \text { is } 1
\end{array}\right. \\
& v_{h_{j}}^{1 s} \equiv\left\{\begin{array}{lll}
0 & \text { if } & s \text {-th component of } j \text { is } 0 \\
q^{s m} \bar{w}^{s}-p^{s} e_{h_{j}}^{s} & \text { if } & s \text {-th component of } j \text { is } 1
\end{array}\right. \\
& N_{t^{0}} \equiv \operatorname{diag}\left(-q^{s m} H_{1}^{s} \bar{w}^{s}-p^{s} \sum_{h_{0} \in \mathcal{H}_{0}^{s}} e_{h_{0}}^{s}\right)_{s=1}^{S} \\
& N_{t^{1}} \equiv \operatorname{diag}\left(q^{s m} H_{1}^{s} \bar{w}^{s}-p^{s} \sum_{h_{1} \in \mathcal{H}_{1}^{s}} p^{s} e_{h_{1}}^{s}\right)_{s=1}^{S}
\end{aligned}
$$

Remark 33 Observe that since

$$
\begin{aligned}
\sum_{j} \sum_{h_{j} \in \mathcal{H}_{j}} v_{h_{j}}^{0 s} & =-\sum_{h^{0} \in \mathcal{H}_{0}^{s}} p^{s} e_{h_{0}}^{s}-H_{1}^{s} q^{s m} \bar{w} \\
\sum_{j} \sum_{h_{j} \in \mathcal{H}_{j}} v_{h_{j}}^{1 s} & =-\sum_{h^{1} \in \mathcal{H}_{1}^{s}} p^{s} e_{h_{1}}^{s}-H_{1}^{s} q^{s m} \bar{w}
\end{aligned}
$$

we have also that

$$
\sum_{j} \sum_{h_{j} \in \mathcal{H}_{j}} \operatorname{diag} v_{h_{j}}^{0}=N_{t_{0}}
$$




$$
\sum_{j} \sum_{h_{j} \in \mathcal{H}_{j}} \operatorname{diag} v_{h_{j}}^{s}=N_{t_{1}}
$$

The above observation allows to sum up the columns of $e_{h}^{\mathbf{1 m}}$ (multiplied by the appropriate expression) to the supercolumns of $t_{0}, t_{1}$ and erase all those columns.

Other symbols.

$$
\widetilde{I} \equiv\left(\begin{array}{cccc}
{\left[0 I_{C-1}\right]} & & & \\
& \ddots & & \\
& & \ddots & \\
& & & {\left[0 I_{C-1}\right]}
\end{array}\right)
$$

for each $h, \quad \lambda_{h}^{1} \equiv\left(\lambda_{h}^{s}\right)_{s=1}^{S}$

\subsection{Proof of Theorem 32}

Define $c \equiv\left(\left(c_{x_{h}}, c_{\lambda_{h}}, c_{b_{h}}, c_{m_{h}^{0}}, c_{\mu_{h}}\right)_{h=1}^{H}, c_{p \backslash}, c_{q}^{m}, c_{q},\left(c_{h}\right)_{h=1}^{H}\right) \in \mathbb{R}^{d i m \Xi+H}$. First of all observe that the desired statement follows if we show that the following system has no solutions.

$$
\left\{\begin{array}{l}
c^{T}\left[D_{\xi, \rho} \widehat{F}_{A}, G_{A}\right]=0 \\
\frac{1}{2} c^{\prime T} c^{\prime}-1=0
\end{array}\right.
$$

where $c^{\prime}=\left(c_{1}, \ldots, c_{H}\right) \in \mathbb{R}^{H}{ }^{20}$. Therefore, it is enough to show that the partial Jacobian of the left hand side of system 25 with respect to $(c, a)$ has full rank. That Jacobian is presented in the table below.

\footnotetext{
${ }^{20}$ Due to generic regularity, in the last equation of system (25), we need to consider only $c^{\prime}$ instead of the all vector $c$.
} 


\begin{tabular}{|c|c|c|c|c|c|c|c|c|c|c|c|c|c|c|c|c|c|}
\hline$\frac{\pi}{4}$ & & & & $2^{\frac{\pi}{8}}$ & & & & & & & & & & & & & \\
\hline$\vec{T}$ & $z^{8}$ & & & & & & & & & & & & & & & & \\
\hline J & & & & $\begin{array}{l}7 \\
y \\
0\end{array}$ & & & & & & & & & & & & & \\
\hline$\vec{u}$ & $\overrightarrow{\vec{z}}$ & & & & & & & & & & & & & & & & \\
\hline $\overrightarrow{E_{0}}$ & & & & & & & $O_{E}^{H}$ & & & & $\xi$ & & 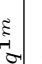 & $\theta$ & & & \\
\hline $\begin{array}{l}\tilde{\xi} \\
0^{\circ}\end{array}$ & & & & & & & & & & & -1 & & -1 & & 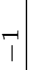 & $\overrightarrow{1}$ & \\
\hline $0^{2 x}$ & & & & $\partial_{i}^{t}$ & & & $0 \frac{H}{N}$ & & & & & 웟 & है & $\left|\frac{2}{2}\right|$ & & & \\
\hline is & $0^{t} \theta$ & & & & & & $0 \frac{H}{N}$ & & & 복 & $\overrightarrow{E_{0}}$ & & & $|\vec{z}|$ & & & \\
\hline $0^{2 x}$ & & & & $\mathrm{~s}_{2}$ & & & 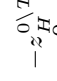 & i & 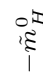 & & & & 菅 & & & 芯 & \\
\hline $0^{0-1}$ & $\mathrm{H}_{a}$ & & & & & & $\begin{array}{c}1 \\
1 \\
0 \\
0\end{array}$ & & 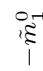 & & $\begin{array}{l}\tilde{\Xi} \\
0 \\
1\end{array}$ & & & & 芯 & & \\
\hline$\sigma$ & & & & & & & & & & -1 & & - & & & & & \\
\hline $0^{\overline{2}}$ & E & & & 吢 & & & & & & & & & & & & & \\
\hline $0_{0}^{\mathbb{I}}$ & & & & & & -1 & & & & & & & & & & & \\
\hline $\begin{array}{l}0^{2} \\
0^{E}\end{array}$ & & & & & 㤐 & -1 & & & $0^{2}$ & & & & & 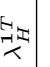 & & & \\
\hline $0^{\pi}$ & & & & & 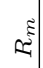 & & & 年 & & & & & & $\lambda^{2}$ & & & \\
\hline$\frac{\pi}{3}$ & & & & 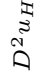 & $\theta$ & & $\underbrace{+4}_{1}$ & & & & & & & & & & \\
\hline $0_{0}^{5}$ & & & & & & & & & & & -1 & & & & & & \\
\hline है & & 㤐 & $\rightarrow$ & & & & & & $\stackrel{0-1}{x}$ & & & & & $\mathrm{E}_{x}$ & & & \\
\hline 8 & & |f & & & & & & 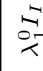 & & & & & & $\lambda^{2}$ & & & \\
\hline 8 & $\begin{array}{l}\overrightarrow{3} \\
\text { ปี }\end{array}$ & $\begin{array}{l}\theta \\
1\end{array}$ & & & & & $\stackrel{t \rightarrow r}{<}$ & & & & & & & & & & \\
\hline & $\stackrel{-}{\rightarrow}$ & $\stackrel{\sim}{-}$ & $\stackrel{10}{-}$ & \pm & $\stackrel{N}{ \pm}$ & $\stackrel{10}{20}$ & 0 & $r$ & $\infty$ & $\stackrel{\infty}{-}$ & $\stackrel{\vec{H}}{\rightarrow}$ & $\stackrel{m}{ \pm}$ & \pm & o & $c$ & \pm & $\exists$ \\
\hline
\end{tabular}


In the first column of the table, we put the names of the corresponding row of the Jacobian; in the first row of the table, the variables we differentiate with respect to. In fact, for simplicity, we consider just two households (household 1 and $H$ ) and we reorder the rows of the Jacobian under analysis to make more transparent the rank computation described in Case 1 below. For each $h, N\left(c_{x_{h}}\right)$ is the partial Jacobian of the left hand side of the subsystem of system (25) obtained multiplying $c^{T}$ times the partial Jacobian of the function $\left(\widehat{F}_{A}, G_{A}\right)$ with respect to $x_{h}$. Since $N\left(c_{x_{h}}\right)$ has full rank if and only if $c_{x_{h}} \neq 0$, we have to distinguish some cases with respect to the values of $\left(c_{x_{h}}\right)_{h=1}^{H}$ :

Case 1. For each $h, c_{x_{h}} \neq 0$; Case 2. For each $h, c_{x_{h}}=0$; Case 3. There exist $h, h^{\prime}$ such that $c_{x_{h}} \neq 0$ and $c_{x_{h^{\prime}}}=0$.

Case 1. For each $h, c_{x_{h}} \neq 0$.

In this case, we show that condition 23 holds. Preliminary observe that the partial Jacobian of the left hand side of equations $\left[(h .3)_{h=1}^{H},(h .4)_{h=1}^{H},(9)\right]$ with respect to $\left(\left(c_{\lambda_{h}}\right)_{h=1}^{H}, c_{q m}\right)$ has full rank. We can then use the so called perturbation method - see for example Villanacci and others (2002) - to show that matrix (26) has full rank ${ }^{21}$.

- $(1.1) \leftarrow a_{1}$.

- $(1.2) \leftarrow\left(c_{x_{1}^{s 1}}\right)_{s=0}^{S} \rightsquigarrow(1.1)$;

$(1.1) \leftarrow a_{1}$.

- $(1.5) \leftarrow c_{m_{1}^{0}} \rightsquigarrow(1.2)$;

$(1.2) \leftarrow\left(c_{x_{1}^{s 1}}\right)_{s=0}^{S} \rightsquigarrow(1.1)$;

$(1.1) \leftarrow a_{1}$.

- $(H .1) \leftarrow a_{H}$.

- $(H .2) \leftarrow\left(c_{x_{H}^{s 1}}\right)_{s=0}^{S} \rightsquigarrow(H .1)$;

$(H .1) \leftarrow a_{H}$.

- $(H .5) \leftarrow c_{\mu_{H}}$.

- $\left(6^{\backslash}\right) \leftarrow c_{x_{h}}=\left(c_{x_{h}^{s c}}\right)_{c \neq 1} \rightsquigarrow(h .1),(h .2)$, for some $h$;

$(h .2) \leftarrow\left(c_{x_{h}^{s 1}}\right)_{s=0}^{S} \rightsquigarrow(h .1) ;$

$(h .1) \leftarrow a_{H}$.

\footnotetext{
${ }^{21}$ That method is nothing but a riformulation of the fact that elementary column and row operations do not affect the rank of a matrix.
} 


$$
\begin{aligned}
& \text { • }(7),(8)] \leftarrow\left(c_{b_{H}}, c_{m_{H}^{0}}\right) \rightsquigarrow(H .5),(H .2) ; \\
&(H .5) \leftarrow c_{\mu_{H}} ; \\
&(H .2) \leftarrow\left(c_{x_{H}^{s 1}}\right)_{s=0}^{S} \rightsquigarrow(H .1) ; \\
&(H .1) \leftarrow a_{H} . \\
& \text { - }\left[(h .3)_{h=1}^{H},(h .4)_{h=1}^{H},(9)\right] \leftarrow\left(c_{\lambda_{h}^{1}}\right)_{h=1}^{H}, c_{q^{0 m}}, c_{q^{m 1}} \rightsquigarrow(h .1)_{h=1}^{H},(6 \backslash) ; \\
&(6 \backslash) \leftarrow c_{x_{1}^{\backslash}}=\left(c_{x_{1}^{s c}}\right)_{c \neq 1} \rightsquigarrow(1.1),(1.2) ; \\
&(1.2) \leftarrow\left(c_{x_{1}^{s, 1}}\right)_{s=0}^{S} \rightsquigarrow(1.1) ; \\
&(h .1)_{h=1}^{H} \leftarrow\left(a_{h}\right)_{h=1}^{H} \cdot \\
& \text { - }(10) \leftarrow\left(c_{\lambda_{h}^{0}}\right)_{h=1}^{H} \rightsquigarrow(h .1)_{h=1}^{H},(6),(7),(8),\left[(h .3)_{h=1}^{H},(h .4)_{h=1}^{H},(9)\right] ; \\
&-[(7),(8)] \leftarrow\left(c_{b_{H}}, c_{m_{H}^{0}}\right) \rightsquigarrow(H .5),(H .2),(9) ; \\
& \quad(H .5) \leftarrow c_{\mu_{H}} ; \\
& \quad(H .2) \leftarrow\left(c_{x_{H}^{s, 1}}\right)_{s=0}^{S} \rightsquigarrow(H .1) ; \\
&-\left[(h .3)_{h=1}^{H},(h .4)_{h=1}^{H},(9)\right] \leftarrow\left(c_{\lambda_{h}^{1}}\right)_{h=1}^{H}, c_{q}, c_{q^{0 m}}, c_{q^{m 1}} \rightsquigarrow(h .1)_{h=1}^{H},(6 \backslash) ; \\
& \quad(6 \backslash) \leftarrow c_{x_{h}^{\backslash}}=\left(c_{x_{h}^{s c}}\right)_{c \neq 1, s=0}^{S} \rightsquigarrow(h .1),(h .2), \text { for some } h ; \\
& \quad(h .2) \leftarrow\left(c_{x_{h}^{s 1}}\right)_{s=0}^{S} \rightsquigarrow(h .1) ; \\
&-(h .1)_{h=1}^{H} \leftarrow\left(a_{h}\right)_{h=1}^{H} .
\end{aligned}
$$

Since $\frac{1}{2} c^{\prime} c^{\prime}=1$, there is $\bar{h} \leq H$ such that $c_{\bar{h}} \neq 0$. Therefore,

- $(11) \leftarrow c_{\bar{h}} \rightsquigarrow(\bar{h} \cdot 1)$;

$$
(\bar{h} .1) \leftarrow a_{\bar{h}} .
$$

Case 2. For each $h, c_{x_{h}}=0$.

In this case, we are going to show that Condition 22 holds. Substituting in matrix 25 the values of $c_{x_{h}}$ and using first order conditions of the households' problems we get a new system. It is in fact enough to consider a subsystem of that one whose Jacobian is presented below; in the first column, we list the names of equations and in the first rows, the variables we take derivatives with respect to. 


\begin{tabular}{|c|c|c|c|c|c|c|c|c|c|c|c|}
\hline & $\begin{array}{c}S+1 \rightarrow \\
c_{\lambda_{1}^{0}}+1 \\
\end{array}$ & $c_{\lambda_{1}^{1}}$ & $\cdot c_{\lambda_{1}^{S}}^{\cdot}$ & $\begin{array}{c}S_{C_{\lambda}^{0}+1 \rightarrow}^{+1 \rightarrow} \\
{ }_{H}\end{array}$ & $c_{\lambda_{H}^{1}}^{\cdot}$ & $c_{\lambda_{H}^{S}}$ & $\begin{array}{l}S+1 \rightarrow \rightarrow \\
{ }_{q^{0 m}}^{+1 m}\end{array}$ & ${ }^{c} q^{1 m}$ & $c_{q} \dot{S} m$ & ${ }_{c_{1}}^{H}$ & ${ }^{c}{ }_{H}$ \\
\hline \multirow[t]{4}{*}{$\begin{array}{c}(1.1 \\
\text { new }) \\
S+1\end{array}$} & -1 & & & & & & & & & $\lambda_{1}^{0}$ & \\
\hline & & -1 & & & & & & & & $\lambda_{1}^{1}$ & \\
\hline & & & & & & & & & & $\ldots$ & \\
\hline & & & -1 & & & & & & & $\lambda_{1}^{S}$ & \\
\hline \multirow[t]{4}{*}{$\begin{array}{c}(H .1 \\
n e w) \\
S+1\end{array}$} & & & & -1 & & & & & & & $\lambda_{H}^{0}$ \\
\hline & & & & & -1 & & & & & & $\lambda_{H}^{1}$ \\
\hline & & & & & & & & & & & $\ldots$ \\
\hline & & & & & & -1 & & & & & $\lambda_{H}^{S}$ \\
\hline $\begin{array}{c}(6.1 .2) \\
S \downarrow\end{array}$ & & $-z_{1}^{12}$ & & & $-\tilde{z}_{H}^{12}$ & & & $-\sigma^{12}$ & & & \\
\hline$(6 . S .2)$ & & & $-\tilde{z}_{1}^{S 2}$ & & & $-\widetilde{z}_{H}^{S 2}$ & & & $-\sigma S 2$ & & \\
\hline $\begin{array}{c}(H .4) \\
1\end{array}$ & & & & $-q^{m 0}$ & $q^{1 m}$ & $q^{S m}$ & 1 & $q^{1 m}$ & $q^{S m}$ & & \\
\hline $\begin{array}{c}(10.1) \\
H \downarrow\end{array}$ & $q^{0 m}$ & & & & & & -1 & & & & \\
\hline$(10 . H)$ & & & & $q^{0 m}$ & & & -1 & & & & \\
\hline
\end{tabular}

where by (6new) we mean equations $(6 . s .2)_{s=1}^{S}$ in equations (6). Observe that the above subsystem of system (19) is a system with the same number of equations and unknowns, in fact, $H(S+1)+S+1+H$. We want to show that $\left(\left(c_{\lambda_{h}}, c_{h}\right)_{h=1}^{H}, c_{q^{m}}\right)=0$ is the only solution of the above system. Therefore, from $\left(c_{\lambda_{1}}, c_{q^{m}}\right)=0$ and from equation (1.4) we have $c_{\mu_{1}}=0$ and then $c=0$, contradicting equation (11) in system (19).

Performing some elementary columns and row operations on the Jacobian presented above, it is possible to show that its determinant is

$$
\begin{aligned}
& \gamma_{1}\left(q^{m}, t,\left(x_{h}^{s 2}, e_{h}^{s 2}\right)_{\substack{s=1, \ldots, S \\
h=1, ., H}}\right) \equiv 1-\sum_{s} \frac{q^{s m}}{\sigma^{s 2}} \sum_{h} \frac{\lambda_{h}^{s} \widetilde{z}_{h}^{s 2}}{\lambda_{h}^{0} q^{0 m}} \equiv \\
& =1-\sum_{s} \frac{q^{s m}}{q^{0 m}} \frac{\lambda_{h_{0} \in \mathcal{H}_{0}} t_{0}^{s} e_{h_{0}}^{s 2}+\sum_{h_{1} \in \mathcal{H}_{1}} t_{1}^{s} e_{h_{1}}^{s 2}}{\sum_{0}} \\
& \cdot\left(\sum_{h_{0} \in \mathcal{H}_{0}} \frac{\lambda_{h_{0}}^{s}}{\lambda_{h_{0}}^{0}}\left(x_{h_{0}}^{s 2}-e_{h_{0}}^{s 2}+t_{0}^{s} e_{h_{0}}^{s 2}\right)+\sum_{h_{1} \in \mathcal{H}_{1}} \frac{\lambda_{h_{1}}^{s}}{\lambda_{h_{1}}^{0}}\left(x_{h_{1}}^{s 2}-e_{h_{1}}^{s 2}+t_{1}^{s} e_{h_{1}}^{s 2}\right)\right)
\end{aligned}
$$

which is shown to be different from zero in Lemma 31.

Case 3. There exist $h, h^{\prime}$ such that $c_{x_{h}} \neq 0$ and $c_{x_{h^{\prime}}}=0$.

In this case, we are going to use the following strategy. We first substitute $c_{x_{h}}=0$ in system (19). We then disregard some equations, being sure that their number is still bigger than the number of unknown. We then show that 
generically the new system has no solution, using transversality in a way which is similar to that one used in Case 1.

\section{References}

Carosi L. (2001), Optimality in a financial economy with restricted participation, Decisions in Economics and Finance, 24, 1-19.

Cass D. (1992), Incomplete Financial Markets and Indeterminacy of Competitive Equilibrium, in J. J. Laffont ed., Advances in Economic Theory; Sixth World Congress, vol. 2, Cambridge University Press, Cambridge, 262-88.

Cass, D. and Shell K. (1980), In Defense of a Basic Approach, in Models of Monetary Economics, J.H. Kareken and N. Wallace eds., Minneapolis, Federal Reserve Bank of Minneapolis.

Cass D., Siconolfi. P, and Villanacci, A., (2001), Generic Regularity of Competitive Equilibria with Restricted Participation, Journal of Mathematical Economics, 36, 61-76.

Citanna A., Kajii A., and Villanacci A. (1998), Constrained suboptimality in incomplete markets: a general approach and two applications, Economic Theory, Mini-Symposium on Pareto Improvement in Incomplete Financial Markets, Economic Theory 11, 495-521.

Citanna A., Polemarchakis, H. and Tirelli, M., (2002), "The Taxation of Trades in Assets", CORE-Universitè Catholique de Louvaine.

Clower, R. W., (1967), A reconsiderations of the Microfoundations of Monetary Theory, Western Economic Journal 6, 1-9.

del Mercato, E. L., Villanacci, A., (2003), Taxes and Outside Money in Incomplete Financial Markets: the case of proportional taxes, mimeo.

del Mercato, E. L., and Villanacci, A., (in progress), Cash-in-Advance Models and Pareto Improvements in Incomplete Financial Markets, mimeo.

Geanakoplos J., and Polemarchakis H. M. (1986), Existence, regularity, and constrained suboptimality of competitive allocations when assets structure is incomplete, in W. P. Heller, R. M. Starr, and D. A. Starret Eds, Essays in honor of K. J. Arrow, Cambridge University Press, Cambridge, Vol 3, 65-95.

Knapp, G. F., (1905), Die Staatliche Theorie des Geldes, Munich, Duncker and Humblot. English translation: The State Theory of Money, London, Macmillan, (1924).

Hirsch M. (1976), Differential Topology, Springer-Verlag, New York, NY, 1976.

Lerner, A. P., 1947, Money as a Creature of the State, American Economic Review 37, 312-317. 
Magill M., and Quinzii M. (1992), Real Effects of Money in General Equilibrium, Journal of Mathematical Economics, 21, 301-42.

Magill M., and Quinzii M. (1996), Theory of Incomplete Markets, vol. I, The MIT Press, Cambridge, Massachusetts.

Starr, R.M., (1989), Introduction, in: R.M.Starr eds., General Equilibrium models of monetary economies. Studies in Static Foundations of Monetary Theory, Academic Press Inc., San Diego.

Villanacci A. (1991), Real Indeterminacy, Taxes and Outside Money in Incomplete Financial Market Economies: I. The case of Lump Sum Taxes, in M. Galeotti, L. Geronazzo, F. Gori, eds., Proceedings of the Second Meeting on Non-linear Dynamics in Economics, Springer Verlag.

Villanacci A., (1993), Real Indeterminacy, Taxes and Outside Money in Incomplete Financial Market Economies: II. The Case of Taxes as Function of Wealth, DIMADEFAS Working Paper, University of Florence, Italy.

Villanacci, A., L. Carosi, P. Benevieri and A. Battinelli, (2002) Differential Topology and General Equilibrium with Complete and Incomplete Markets, Kluwer Academic Publishers. 